\title{
VISCOSITY SOLUTIONS TO PARABOLIC COMPLEX MONGE-AMPÈRE EQUATIONS
}

\author{
HOANG-SON DO, GIANG LE, AND TAT DAT TÔ
}

\begin{abstract}
In this paper, we study the Cauchy-Dirichlet problem for Parabolic complex Monge-Ampère equations on a strongly pseudoconvex domain using the viscosity method. We extend the results in [EGZ15b] on the existence of solution and the convergence at infinity. We also establish the Hölder regularity of the solutions when the Cauchy-Dirichlet data are Hölder continuous.
\end{abstract}

\section{INTRODUCTION}

In [ST17, ST12], Song and Tian gave a conjectural picture to approach the Minimal Model Program via the Kähler-Ricci flow. In the Song-Tian program, one need to study the behavior of the Kähler-Ricci flow on mildly singular varieties. This requires a theory of weak solutions for certain degenerate parabolic complex Monge-Ampère equations modelled on

$$
\left(d d^{c} u\right)^{n}=e^{\partial_{t} u(t, z)+F(t, z, u)} \mu,
$$

where $\mu$ is a volume form, and $u$ is $t$-dependent Kähler potential on a compact Kähler manifold.

A viscosity approach for complex elliptic Monge-Ampère equations was established in [EGZ11, EGZ15a, Wan12, HL09] (see also [DDT19] for a recent generalization). In [EGZ11, EGZ15a], Eyssidieux-Guedj-Zeriahi modified the arguments in [IL90, CIL92] to prove local and global comparison principles and solve complex Monge-Ampère equations on both compact Kähler manifolds and complex domains. They also compared the viscosity solutions and the plurisubharmonic solutions. On the other hand, using the contact set techniques (the AlexandrovBakelmanPucci estimate), Wang [Wan12] also proved a local viscosity comparison principle and applied to the Dirichlet problem for Monge-Ampère equations without using the argument in [IL90, CIL92]. In [HL09], Harvey and Lawson also used the viscosity method to the Dirichlet problem for the homogeneous complex Monge-Ampère equation on smooth hyperconvex domains in Stein manifolds.

A complementary viscosity approach for degenerate parabolic Monge-Ampère equations has been developed recently by Eyssidieux-Guedj-Zeriahi [EGZ15b] in domains of $\mathbb{C}^{n}$ and [EGZ16, EGZ18] on compact Kähler manifolds (see also [Tô19] for a generalization). This approach also adapted the viscosity approach developped by Lions et al. (see [IL90, CIL92]) to the complex case, using the elliptic side of the theory which was developed in [EGZ11].

Date: November 26, 2019

The first-named author was funded by the Vietnam National Foundation for Science and Technology Development (NAFOSTED) under grant number 101.02-2017.306.

Keywords: Viscosity solutions, Parabolic Monge-Ampère equation, pluripotential theory. 
In [EGZ15b], Eyssidieux-Guedj-Zeriahi studied a Cauchy-Dirichlet problem for (1) in which the density $\mu$ and parabolic boundary condition are independent of time. They proved that in this case the Cauchy-Dirichlet problem for (1) admits a solution if the problem is admissible (see below).

In this note, we solve a more general Cauchy-Dirichlet problem on a pseudoconvex domain for (1) in which the density $\mu$ and the parabolic boundary condition depend on time. We also analyzed the solvability of the Cauchy-Dirichlet problem by giving several characterizations for the admissible condition. In addition, we establish the Hölder regularity of the solutions. Finally we prove that the solution of the Cauchy-Dirichlet problem converges, as $t \rightarrow \infty$, to the solution of the corresponding Dirichlet problem, extending the convergence result in [EGZ15b].

There is a well established pluripotential theory of weak solutions to elliptic complex Monge-Ampère equations, following the pionneering work of Bedford and Taylor [BT76, BT82] in local case, but the similar theory for the parabolic side only developed recently [GLZ1, GLZ2]. It is very interesting to compare the viscosity and pluripotential concepts, this requires Theorem 1.2 below. We refer the reader to [GLZ3] for more details.

We now explain the precise context. Let $\Omega \subset \mathbb{C}^{n}$ be a smooth bounded strongly pseudoconvex domain and $T \in(0, \infty)$. We consider the Cauchy-Dirichlet problem

$$
\left\{\begin{array}{l}
e^{\partial_{t} u+F(t, z, u)} \mu(t, z)=\left(d d^{c} u\right)^{n} \quad \text { in } \quad \Omega_{T}, \\
u=\varphi \quad \text { in } \quad[0, T) \times \partial \Omega, \\
u(0, z)=u_{0}(z) \quad \text { in } \bar{\Omega},
\end{array}\right.
$$

where

- $\Omega_{T}=(0, T) \times \Omega$.

- $F(t, z, r)$ is continuous in $[0, T] \times \bar{\Omega} \times \mathbb{R}$ and non-decreasing in $r$.

- $\mu(t, z)=f(t, z) d V$, where $d V$ is the standard volume form in $\mathbb{C}^{n}$ and $f \geq 0$ is a bounded continuous function in $[0, T] \times \Omega$.

- $\varphi(t, z)$ is a continuous function in $[0, T] \times \partial \Omega$.

- $u_{0}(z)$ is continuous in $\bar{\Omega}$ and plurisubharmonic in $\Omega$ such that $u_{0}(z)=\varphi(0, z)$ in $\partial \Omega$.

By [EGZ15b, Definition 5.6], $\left(u_{0}, \mu(0,).\right)$ is called admissible if for all $\epsilon>0$, there exists $u_{\epsilon} \in P S H(\Omega) \cap C(\bar{\Omega})$ and $C_{\epsilon}>0$ such that $u_{0} \leq u_{\epsilon} \leq u_{0}+\epsilon$ and $\left(d d^{c} u_{\epsilon}\right)^{n} \leq e^{C_{\epsilon}} \mu(0, z)$ in the viscosity sense. We observe below that the condition $u_{\epsilon} \in P S H(\Omega)$ is redundant (see Theorem 1.3 (i)). We still use the admissible term for the same definition above without this condition.

Definition 1.1. We say that $\left(u_{0}, \mu(0,).\right)$ is admissible if for all $\epsilon>0$, there exist $u_{\epsilon} \in C(\bar{\Omega})$ and $C_{\epsilon}>0$ such that $u_{0} \leq u_{\epsilon} \leq u_{0}+\epsilon$ and $\left(d d^{c} u_{\epsilon}\right)^{n} \leq e^{C_{\epsilon}} \mu(0, z)$ in the viscosity sense.

It follows from [EGZ15b] that if $\varphi, \mu$ are independent of $t$ and $\left(u_{0}, \mu\right)$ is admissible then (2) admits a solution. In this paper, we extend this result to the case in which $\varphi$ and $\mu$ depend on $t$ as well. Our first main result is the following

Theorem 1.2. Assume that $\mu=f d V$ where $f$ is independent of $t$. If $\left(u_{0}(z), \mu\right)$ is admissible then the equation (2) admits a unique solution. 
Although $F$ and $f$ play different roles, they are not entirely disjoint. If $f \in$ $C([0, T] \times \bar{\Omega})$ and $f>0$ then the problem can be reduced to the case $f=1$. If $f \in C([0, T] \times \bar{\Omega})$ and $\frac{f(t, z)}{f(0, z)}$ can be extended to a continuous positive function on $[0, T] \times \bar{\Omega}$ then the problem can be reduced to the case where $f$ is independent of $t$. In this paper, we also obtain the existence result to certain cases where $f$ depends on $t$ as well. Moreover, the set $\{z: f(t, z)=0\}$ may also depend on $t$ (and then the problem can not be reduced to the case where $f$ is independent of $t$ ). We refer to Theorem 4.13 and Corollary 4.14 for more details.

We are now interested in the notion of admissible data. In order to avoid confusion, we always denote by $\left(d d^{c} \phi\right)_{P}^{n}$ the Monge-Ampère measure in the pluripotential sense (see [BT76, BT82, Ceg04, Blo06] for the definition) of a psh function $\phi$ if this measure is well-defined. We obtain the following properties:

Theorem 1.3. Let $g \geq 0$ be a bounded continuous function in $\Omega$ and $\nu=g d V$. Let $\phi \in P S H(\Omega) \cap C(\bar{\Omega})$. Then the following holds:

(i) If $(\phi, \nu)$ is admissible then the function $u_{\epsilon}$ in the definition 1.1 can be taken to be psh in $\Omega$.

(ii) Admissibility is a local property: if for every $z \in \Omega$, there exists an open neighborhood $U$ of $z$ such that $(\phi, \nu)$ is admissible in $U$ then $(\phi, \nu)$ is admissible in $\Omega$.

(iii) If $\int_{\{g=0\}}\left(d d^{c} \phi\right)_{P}^{n}=0$ then $(\phi, \nu)$ is admissible.

In particular, when $\mu$ is independent of time, we prove that this condition is also necessary (Corollary 3.6 and Remark 4.11). However, we also give a counterexample in which $\mu$ depends on $t$, the Cauchy-Dirichlet problem admits a solution but $\left(u_{0}, \mu(0, z)\right)$ is not admissible. In addition, we prove the following local and integral criteria to the admissible condition.

Corollary 1.4. If $\nu=g(z) d V \geq 0$ with $\{z \in \Omega: g(z)=0\}$ is a negligible set, then $(\phi, \nu)$ is admissible for every $\phi \in C(\bar{\Omega}) \cap P S H(\Omega)$.

For degenerate (elliptic) complex Monge-Ampère equations, the Hölder regularity of pluripotential solutions has been studied intensively (we refer to [GKZ08, DDGHKZ] and references therein). Similar results for viscosity solutions can be found in [Lu13, Wan12]. Nevertheless, to the best of our knowledge, no Hölder regularity result to the weak solutions of parabolic complex Monge-Ampère equations has been established in both pluripotential and viscosity senses (in the non-smooth case). In this paper, we make a first step in this direction:

Theorem 1.5. Assume that $\mu=d V$ and $u(t, z)$ is a viscosity solution to (2). Suppose that there exist $C>0,0<\alpha<1$ and $0<\beta<1 / 2$ such that

$$
\begin{gathered}
|\varphi(t, z)-\varphi(s, w)| \leq C\left(|t-s|^{\alpha}+|z-w|^{2 \beta}\right), \forall z, w \in \partial \Omega, t, s \in[0, T), \\
\varphi(t, z)-\varphi(s, z) \leq C(t-s), \forall z \in \partial \Omega, 0<s<t<T,
\end{gathered}
$$

and

$$
\left|u_{0}(z)-u_{0}(w)\right| \leq C|z-w|^{\beta}, \forall z, w \in \bar{\Omega} .
$$

Suppose also that, for any $K>0$, there exists $C_{K}>0$ such that,

$$
|F(t, z, r)-F(t, w, r)| \leq C_{K}|z-w|^{\beta} \text {. }
$$


for all $z, w \in \Omega, t \in[0, T), r \in[-K, K]$. Then, there exists $\tilde{C}>0$ such that

$$
|u(t, z)-u(s, w)| \leq \tilde{C}\left(|t-s|^{\alpha}+|z-w|^{\beta}\right),
$$

for all $z, w \in \Omega, t, s \in[0, T)$.

Moreover, if $\varphi$ is Lipschitz in $t$ then $u$ is locally Lipschitz in $t$ uniformly in $z$.

Finally, we prove that the viscosity solution of the Cauchy-Dirichlet problem (2) asymptotically recovers the solution of the corresponding elliptic Dirichlet problem under some assumptions. This also extends the convergence result in [EGZ15b].

Theorem 1.6. Assume that $T=\infty, \varphi(t, z) \rightrightarrows \varphi_{\infty}(z)$ as $t \rightarrow \infty$ and $F(t, z, r) \rightrightarrows$ $F_{\infty}(z, r)$ in $\bar{\Omega} \times \mathbb{R}$ as $t \rightarrow \infty$, where $\rightrightarrows$ denotes the uniform convergence.

Suppose that $\sup _{t>0} f(t, z) \in L^{p}(\Omega)$ for some $p>1$ and $f(t, z)$ converges almost everywhere to a function $f_{\infty}(z)$ as $t \rightarrow \infty$. Then $u(t, z)$ converges uniformly to $u_{\infty}(z)$ as $t \rightarrow \infty$, where $u_{\infty}$ is the unique solution of the equation

$$
\left\{\begin{array}{l}
u_{\infty} \in P S H(\Omega) \cap C(\bar{\Omega}) \\
\left(d d^{c} u_{\infty}\right)_{P}^{n}=e^{F_{\infty}\left(z, u_{\infty}\right)} f_{\infty}(z) d V(z) \quad \text { in } \quad \Omega \\
u_{\infty}=\varphi_{\infty} \quad \text { in } \quad \partial \Omega .
\end{array}\right.
$$

The solution $u_{\infty}$ to the elliptic Dirichlet problem above is well known to exist in the pluripotential sense in [Kol98]. If $f_{\infty}$ is continuous then the solution in the pluripotential sense is also the solution in the viscosity sense [EGZ11, HL09, Wan12].

In fact, we can also obtain the uniform convergence in capacity when $p=1$ as well, we refer to Theorem 6.1. In this case, the equation (3) is replaced by the equation (61). The existence of the pluripotential solution to (61) holds due to [Ceg04], [Aha07], [ACCP09] (see also 2.17).

Acknowledgement. The authors are grateful to Vincent Guedj and Ahmed Zeriahi for very useful discussions. This work was begun during a visit by the first-named author to the Institut de Mathématiques de Toulouse (from September 1 to September 30, 2018) funded by LIA Formath Vietnam and ANR GRACK. This paper was partially written while the first-named author visited Vietnam Institute for Advanced Study in Mathematics(VIASM). He would like to thank these institutions for their hospitality. The authors would like to thank the referee for very useful comments and suggestions.

\section{Preliminaries}

For the reader's convenience, we recall some basic concepts and well-known results.

2.1. Viscosity concepts. Consider the following parabolic complex Monge-Ampère equations on a bounded domain $\Omega \subset \mathbb{C}^{n}$

$$
e^{\partial_{t} u+F(t, z, u)} \mu(t, z)=\left(d d^{c} u\right)^{n},
$$

where

- $\Omega_{T}=(0, T) \times \Omega$.

- $F(t, z, r)$ is continuous in $[0, T] \times \bar{\Omega} \times \mathbb{R}$ and non-decreasing in $r$.

- $\mu(t, z)=f(t, z) d V$, where $d V$ is the standard volume form in $\mathbb{C}^{n}$ and $f \geq 0$ is a bounded continuous function in $[0, T] \times \Omega$. 
Definition 2.1. (Test functions) Let $w: \Omega_{T} \longrightarrow \mathbb{R}$ be any function defined in $\Omega_{T}$ and $\left(t_{0}, z_{0}\right) \in \Omega_{T}$ a given point. An upper test function (resp. a lower test function) for $w$ at the point $\left(t_{0}, z_{0}\right)$ is a $C^{(1,2)}$-smooth function $q$ (i.e., $q$ is $C^{1}$ in $t$ and $C^{2}$ in $z)$ in a neighbourhood of the point $\left(t_{0}, z_{0}\right)$ such that $w\left(t_{0}, z_{0}\right)=q\left(t_{0}, z_{0}\right)$ and $w \leq q$ (resp. $w \geq q$ ) in a neighbourhood of $\left(t_{0}, z_{0}\right)$. We will write for short $w \leq_{\left(t_{0}, z_{0}\right)} q$ (resp. $\left.w \geq_{\left(t_{0}, z_{0}\right)} q\right)$.

Definition 2.2. 1. A function $u:[0, T) \times \bar{\Omega} \longrightarrow \mathbb{R}$ is said to be a (viscosity) subsolution to the parabolic complex Monge-Ampère equation (4) in $(0, T) \times \Omega$ if $u$ is upper semi-continuous in $[0, T) \times \bar{\Omega}$ and for any point $\left(t_{0}, z_{0}\right) \in \Omega_{T}:=(0, T) \times \Omega$ and any upper test function $q$ for $u$ at $\left(t_{0}, z_{0}\right)$, we have

$$
\left(d d^{c} q_{t_{0}}\left(z_{0}\right)\right)^{n} \geq e^{\partial_{t} q\left(t_{0}, z_{0}\right)+F\left(t_{0}, z_{0}, q\left(t_{0}, z_{0}\right)\right)} \mu\left(t_{0}, z_{0}\right) .
$$

In this case, we also say that $u$ satisfies the differential inequality

$$
\left(d d^{c} u\right)^{n} \geq e^{\partial_{t} u(t, z)+F(t, z, u(t, z))} \mu(t, z),
$$

in the viscosity sense in $\Omega_{T}$.

The function $u$ is called a subsolution to the Cauchy-Dirichlet problem (2) if $u$ is a subsolution to (4) satisfying $u \leq \varphi$ in $[0, T) \times \partial \Omega$ and $u(0, z) \leq u_{0}(z)$ for all $z \in \Omega$.

2. A function $v:[0, T) \times \bar{\Omega} \longrightarrow \mathbb{R}$ is said to be a (viscosity) supersolution to the parabolic complex Monge-Ampère equation (4) in $\Omega_{T}$ if $v$ is lower semi-continuous in $\Omega_{T}$ and for any point $\left(t_{0}, z_{0}\right) \in \Omega_{T}$ and any lower test function $q$ for $v$ at $\left(t_{0}, z_{0}\right)$ such that $d d^{c} q_{t_{0}}\left(z_{0}\right) \geq 0$, we have

$$
\left(d d^{c} q_{t_{0}}\right)^{n}\left(z_{0}\right) \leq e^{\partial_{t} q\left(t_{0}, z_{0}\right)+F\left(t_{0}, z_{0}, q\left(t_{0}, z_{0}\right)\right)} \mu\left(t_{0}, z_{0}\right) .
$$

In this case we also say that $v$ satisfies the differential inequality

$$
\left(d d^{c} v\right)^{n} \leq e^{\partial_{t} v(t, z)+F(t, z, v(t, z))} \mu(t, z),
$$

in the viscosity sense in $\Omega_{T}$.

The function $v$ is called a supersolution to (2) if $v$ is a supersolution to (4) satisfying $v \geq \varphi$ in $[0, T) \times \partial \Omega$ and $v(0, z) \geq u_{0}(z)$ for all $z \in \Omega$.

3. A function $u: \Omega_{T} \longrightarrow \mathbb{R}$ is said to be a (viscosity) solution to the parabolic complex Monge-Ampère equation (4) (respectively, (2)) in $\Omega_{T}$ if it is a subsolution and a supersolution to (4) (respectively, (2)) in $\Omega_{T}$.

Definition 2.3. A discontinuous viscosity solution to the equation (4) (resp. (2)) is a function $u: \Omega_{T} \rightarrow[+\infty,-\infty]$ such that

i) the usc envelope $u^{*}$ of $u$ satisfies $\forall z \in \Omega, u^{*}(t, z)<+\infty$ and is a viscosity subsolution to (4) (resp. (2)),

ii) the lsc envelope $u_{*}$ of $u$ satisfies $\forall z \in \Omega, u_{*}(t, z)>-\infty$ and is a viscosity supersolution to the equation (4) (resp. (2)).

2.2. Basic properties. We recall some basic properties of viscosity subsolution and viscosity supersolution.

Lemma 2.4. Consider the equations

$$
e^{\partial_{t} u+F_{1}(t, z, u)} \mu_{1}(t, z)=\left(d d^{c} u\right)^{n} \quad \text { in } \quad(0, T) \times \Omega
$$


and

$$
e^{\partial_{t} u+F_{2}(t, z, u)} \mu_{2}(t, z)=\left(d d^{c} u\right)^{n} \quad \text { in } \quad(0, T) \times \Omega,
$$

where, for $j=1,2$,

- $F_{j}(t, z, r)$ is continuous in $[0, T) \times \bar{\Omega} \times \mathbb{R}$ and non-decreasing in $r$.

- $\mu_{j}(t, z)=f_{j}(t, z) d V$ with $f_{j}$ is a bounded continuous function in $[0, T) \times \Omega$. Assume that $F_{1} \geq F_{2}$ and $\mu_{1} \geq \mu_{2}$. If $u_{1}$ is a subsolution to (5) then $u_{1}$ is also a subsolution to (6). Conversely, if $u_{2}$ is a supersolution to (6) then $u_{2}$ is also a supersolution to (5).

Lemma 2.5. Let $A>0$. If $u(t, z)$ is a subsolution (resp. supersolution) to (4) in $(0, T) \times \Omega$ then $u_{A}:=\frac{1}{A} u(A t, z)$ is a subsolution (resp. supersolution) to the equation

$$
\frac{1}{A^{n}} e^{\partial_{t} u_{A}+F\left(A t, z, A u_{A}\right)} \mu(A t, z)=\left(d d^{c} u_{A}\right)^{n}
$$

in $\left(0, \frac{T}{A}\right) \times \Omega$.

Lemma 2.6. [CIL92, IS13, EGZ15b] Let $\mu^{j}(t, x) \geq 0$ be a sequence of continuous volume forms converging uniformly to a volume form $\mu$ on $\Omega_{T}$ and let $F^{j}$ be a sequence of continuous functions in $[0, T[\times \Omega \times \mathbb{R}$ converging locally uniformly to a function $F$. Let $\left(u^{j}\right)$ be a locally uniformly bounded sequence of real valued functions defined in $\Omega_{T}$.

1. Assume that for every $j \in \mathbb{N}, u^{j}$ is a viscosity subsolution to the complex Monge-Ampère flow

$$
e^{\partial_{t} u^{j}+F^{j}\left(t, z, u^{j}\right)} \mu^{j}(t, z)-\left(d d^{c} u_{t}^{j}\right)^{n}=0,
$$

associated to $\left(F^{j}, \mu^{j}\right)$ in $\Omega_{T}$. Then its upper relaxed semi-limit

$$
\bar{u}=\limsup _{j \rightarrow+\infty}^{*} u^{j}
$$

of the sequence $\left(u^{j}\right)$ is a subsolution to the parabolic Monge-Ampère equation

$$
e^{\partial_{t} u+F(t, z, u)} \mu-\left(d d^{c} u\right)^{n}=0,
$$

in $\Omega_{T}$.

2. Assume that for every $j \in \mathbb{N}$, $u^{j}$ is a viscosity supersolution to the complex Monge-Ampère flow associated to $\left(F^{j}, \mu^{j}\right)$ in $\Omega_{T}$. Then the lower relaxed semi-limit

$$
\underline{u}=\liminf _{* j \rightarrow+\infty} u^{j}
$$

of the sequence $\left(u^{j}\right)$ is a supersolution to the complex Monge-Ampère flow associated to $(F, \mu)$ in $\Omega_{T}$.

One of applications of Lemma 2.6 is the following

Lemma 2.7. Let $u$ be a subsolution to the equation

$$
e^{\partial_{t} w+F_{1}(t, z, w)} f_{1}(t, z) d V=\left(d d^{c} w\right)^{n},
$$

and $v$ be a supersolution to the equation

$$
e^{\partial_{t} w+F_{2}(t, z, w)} f_{2}(t, z) d V=\left(d d^{c} w\right)^{n},
$$

in $(0, T) \times \Omega$. Let $p$ be a negative plurisubharmonic function in $\Omega$ and $h:(0, T) \rightarrow$ $[0, \infty)$ be a continuous non-decreasing function. Then $\tilde{u}(t, z)=u(t, z)+p(z)-h(t)$ 
is a subsolution to (8) and $\tilde{v}(t, z)=v(t, z)-p(z)+h(t)$ is a supersolution to (9). Moreover, if $p \in \mathcal{E}(\Omega)$ (see Section 2.5 for the definition of the class $\mathcal{E}$ ) and there exist $C_{1}, C_{2}>0$ such that

$$
\partial_{t} u, \partial_{t} v \leq C_{1}
$$

in the viscosity sense and

$$
\sup F(., ., \sup v), \sup F(., ., \sup u) \leq C_{2},
$$

and

$$
\left(d d^{c} p\right)^{n} \geq e^{C_{1}+C_{2}}\left|f_{1}(t, z)-f_{2}(t, z)\right| d V,
$$

in $\Omega$ for every $t \in(0, T)$ then $\tilde{u}$ is a subsolution to (9) and $\tilde{v}$ is a supersolution to $(8)$.

Proof. Let $B \Subset \Omega$ be a ball and $0<a<b<T$. Then, there exist $h_{j}:(a, b) \rightarrow[0, \infty)$ and $p_{j} \in P S H(B) \cap C^{\infty}(\bar{B})$ such that

- $h_{j}$ is smooth and non-decreasing for every $j \in \mathbb{N}$.

- $h_{j} \searrow h$ in $(a, b)$ and $p_{j} \searrow p$ in $\bar{B}$ as $j \rightarrow \infty$.

By the definition of viscosity subsolution and viscosity supersolution, we get $u(t, z)+$ $p_{j}(z)-h_{j}(t)$ is a subsolution to (8) and $v(t, z)-p_{j}(z)+h_{j}(t)$ is a supersolution to $(9)$ in $(a, b) \times B$ for every $j$. Hence, by Lemma 2.6, we have $u(t, z)+p(z)-h(t)$ is a subsolution to $(8)$ and $v(t, z)-p(z)+h(t)$ is a supersolution to $(9)$ in $(a, b) \times B$. Since $a, b$ and $B$ are arbitrary, we obtain the first conclusion.

Now, we assume that (10), (11) and (12) are satisfied. Denote

$$
g(z)=\max _{a \leq t \leq b}\left|f_{1}(t, z)-f_{2}(t, z)\right| .
$$

Then $g \in C(\Omega)$ (by Lemma 2.8) and

$$
\left(d d^{c} p\right)_{P}^{n} \geq e^{C_{1}+C_{2}} g(z) d V,
$$

in $\Omega$. For every $0<\epsilon<d(B, \partial \Omega)$, we denote

$$
g_{\epsilon}(z)=\inf _{|\xi-z|<\epsilon} g(\xi) .
$$

Then by [EGZ11] (pages 1064-1066) and by using convolution, the functions $p_{j}$ can be chosen such that

$$
\left(d d^{c} p_{j}\right)^{n} \geq e^{C_{1}+C_{2}} g_{1 / 2^{j}} d V
$$

in $B$ for every $j$.

Note that $g_{\epsilon}$ converges uniformly to $g$ in $B$ as $\epsilon \searrow 0$. Let $\epsilon_{j} \searrow 0$ such that

$$
\left(d d^{c} \epsilon_{j}|z|^{2}\right)^{n} \geq e^{C_{1}+C_{2}}\left|g_{1 / 2^{j}}(z)-g(z)\right| d V,
$$

in $B$. Denote $q_{j}=p_{j}+\epsilon_{j}|z|^{2}$, we have $q_{j} \in P S H(B) \cap C^{\infty}(\bar{B}), q_{j} \searrow p$ in $\bar{B}$ as $j \rightarrow \infty$ and

$$
\left(d d^{c} q_{j}\right)^{n} \geq e^{C_{1}+C_{2}} g d V .
$$

Then, by the definition, $u(t, z)+q_{j}(z)-h_{j}(t)$ is a subsolution to $(9)$ and $v(t, z)-$ $q_{j}(z)+h_{j}(t)$ is a supersolution to $(8)$ in $(a, b) \times B$ for every $j$.

Hence, by Lemma 2.6, we obtain the second conclusion. 
Lemma 2.8. Assume that $U$ is an open subset of $\mathbb{R}^{m}\left(m \in \mathbb{Z}^{+}\right)$and $a, b$ are real numbers with $a<b$. Suppose that $g:[a, b] \times U \rightarrow \mathbb{R} i s$ a continuous function. Then $h(x)=\sup _{a \leq t \leq b} g(t, x)$ is a continuous function on $U$.

Proof. Since $\{g(t, x)\}_{a \leq t \leq b}$ is a family of continuous functions on $U$, we get that $h$ is lower semi-continuous on $U$. It remains to show that $h$ is upper semi-continuous.

Fix $x_{0} \in U$. Let $V \Subset U$ be an open neighborhood of $x_{0}$. Then, there exists a sequence $\left\{x_{k}\right\}_{k=1}^{\infty} \subset V \backslash\left\{x_{0}\right\}$ such that $x_{k} \stackrel{k \rightarrow \infty}{\longrightarrow} x_{0}$ and $\lim _{k \rightarrow \infty} h\left(x_{k}\right)=\limsup _{x \rightarrow x_{0}} h(x)$.

Since $g\left(t, x_{k}\right)$ is continuous on $[a, b]$, there exists $t_{k} \in[a, b]$ such that $h\left(x_{k}\right)=$ $g\left(t_{k}, x_{k}\right)$. Since $g$ is continuous on the compact set $[a, b] \times \bar{V}$, we have $g$ is uniformly continuous on $[a, b] \times \bar{V}$. Then

$$
\lim _{k \rightarrow \infty}\left(g\left(t_{k}, x_{k}\right)-g\left(t_{k}, x_{0}\right)\right)=0 .
$$

Hence

$$
\lim _{k \rightarrow \infty}\left(h\left(x_{k}\right)-h\left(x_{0}\right)\right)=\lim _{k \rightarrow \infty}\left(g\left(t_{k}, x_{k}\right)-h\left(x_{0}\right)\right) \leq \lim _{k \rightarrow \infty}\left(g\left(t_{k}, x_{k}\right)-g\left(t_{k}, x_{0}\right)\right)=0 .
$$

Thus $h\left(x_{0}\right) \geq \limsup _{x \rightarrow x_{0}} h(x)$. Since $x_{0}$ is arbitrary, we get that $h$ is upper semicontinuous.

The proof is completed.

2.3. Comparison principle and Perron envelope. As is often the case in the viscosity theory and pluripotential theory, one of the main technical tools is the comparison principle:

Theorem 2.9. [EGZ15b] Let u (resp. v) be a bounded subsolution (resp. supersolution) to the parabolic complex MongeAmpère equation (4) in $\Omega_{T}$. Assume that one of the following conditions is satisfied

a) $\mu(t, z)>0$ for every $(t, z) \in(0, T) \times \Omega$.

b) $\mu$ is independent of $t$.

c) Either $u$ or $v$ is locally Lipschitz in $t$ uniformly in $z$.

Then

$$
\sup _{\Omega_{T}}(u-v) \leq \sup _{\partial_{P}\left(\Omega_{T}\right)}(u-v)_{+},
$$

where $u$ (resp. $v$ ) has been extended as an upper (resp. a lower) semicontinuous function to $\overline{\Omega_{T}}$.

Another main technical tool for viscosity theory is Perron method. By using Perron method, one get the following result:

Lemma 2.10. [EGZ15b, Lemma 5.4] Given any non empty family $S_{0}$ of bounded subsolutions to the parabolic equation (4) which is bounded above by a continuous function, the usc regularization of the upper envelope $\phi_{S_{0}}=\sup _{\phi \in S_{0}} \phi$ is a subsolution to (4).

If $S$ is the family of all subsolutions to the Cauchy-Dirichlet problem (2), its envelope $\phi_{S}$ is a discontinuous viscosity solution to (4). 
The above lemma is useful for the case where $\mu(t, z)>0$ or $\mu$ is independent of $t$. In this case, if for every $\epsilon>0$, there exist $\epsilon$-subarriers and $\epsilon$-superbarrier (see the below definition) for (2) then by combining Lemma 2.10 and Theorem 2.9, one obtain the existence of solution to (2).

Definition 2.11. a) A function $u \in U S C([0, T) \times \bar{\Omega})$ is called $\epsilon$-subbarrier for (2) if $u$ is subsolution to (4) in the viscosity sense such that $u_{0}-\epsilon \leq u_{*} \leq u \leq u_{0}$ in $\{0\} \times \bar{\Omega}$ and $\varphi-\epsilon \leq u_{*} \leq u \leq \varphi$ in $[0, T) \times \partial \Omega$.

b) A function $u \in \operatorname{LSC}([0, T) \times \bar{\Omega})$ is called $\epsilon$-superbarrier for (2) if $u$ is supersolution to (4) in the viscosity sense such that $u_{0}+\epsilon \geq u^{*} \geq u \geq u_{0}$ in $\{0\} \times \bar{\Omega}$ and $\varphi+\epsilon \geq u^{*} \geq u \geq \varphi$ in $[0, T) \times \partial \Omega$.

In this paper, we need a modified version of Lemma 2.10:

Lemma 2.12. Assume that for every $\epsilon>0$, the problem (2) admits a continuous $\epsilon$-superbarrier which is Lipschitz in $t$ and a continuous $\epsilon$-subbarrier. Denote by $S$ the family of all continuous subsolutions to (2). Then $\phi_{S}=\sup \{v: v \in S\}$ is a discontinuous viscosity solution to (2).

Proof. By the existence of $\epsilon$-subbarriers and $\epsilon$-superbarriers, and by using Theorem 2.9, we have $\phi_{S}$ satisfies the boundary condition and initial condition of (2). Moreover, the set of continuous points of $\phi_{S}$ contains $\partial_{P} \Omega_{T}$. Hence, $\phi_{S}$ is a discontinuous viscosity solution to (2) iff it is a discontinuous viscosity solution to (4). By Lemma 2.10, we have $\left(\phi_{S}\right)^{*}$ is a subsolution to (4). Then, it remains to show that $\left(\phi_{S}\right)_{*}\left(=\phi_{S}\right)$ is a supersolution to $(4)$.

Assume that $\phi_{S}$ is not a supersolution to (4). Then, there exist a point $\left(t_{0}, z_{0}\right) \in$ $(0, T) \times \Omega$, an open neighborhood $I \times U \Subset(0, T) \times \Omega$ of $\left(t_{0}, z_{0}\right)$ and a $C^{(1,2)}$ function $p: I \times U \rightarrow \mathbb{R}$ such that

- $\left(\phi_{S}-p\right)\left(t_{0}, z_{0}\right)=\min _{I \times U}\left(\phi_{S}-p\right)$;

- At $\left(t_{0}, z_{0}\right)$, the complex Hessian matrix $\left(\frac{\partial^{2} p}{\partial z_{j} \partial \bar{z}_{k}}\right)_{1 \leq j, k \leq n}$ of $p$ is positive and $\left(d d^{c} p\right)^{n}>e^{\partial_{t} p\left(t_{0}, z_{0}\right)+F\left(t_{0}, z_{0}, p\left(t_{0}, z_{0}\right)\right)} \mu\left(t_{0}, z_{0}\right)$.

Let $0<\delta \ll 1$ such that, in a neighborhood $I_{\delta} \times U_{\delta} \subset I \times U$ of $\left(t_{0}, z_{0}\right)$, the functions

$$
p_{\delta}^{ \pm}=p-\delta\left|z-z_{0}\right|^{2} \mp \delta\left(t-t_{0}\right)
$$

are plurisubharmonic in $z$ and

$$
\left(d d^{c} p_{\delta}^{ \pm}\right)^{n}>e^{\partial_{t} p_{\delta}^{ \pm}+F\left(t, z, p_{\delta}^{ \pm}(t, z)\right)} \mu(t, z) .
$$

Let $0<\delta_{1} \ll 1$ such that

$$
\delta_{2}:=-\delta_{1}+\delta \min _{\partial\left(I_{\delta} \times U_{\delta}\right)}\left(\left|t-t_{0}\right|+\left|z-z_{0}\right|^{2}\right)>0 .
$$

We define

$$
\tilde{p}:=\min \left\{p_{\delta}^{+}, p_{\delta}^{-}\right\}+\delta_{1}=p-\delta\left(\left|t-t_{0}\right|+\left|z-z_{0}\right|^{2}\right)+\delta_{1} .
$$

Then $\tilde{p}$ is a continuous subsolution to (4) in $I_{\delta} \times U_{\delta}$ such that $\tilde{p}<\phi_{S}-\delta_{2}$ on $\partial\left(I_{\delta} \times U_{\delta}\right)$ and $\tilde{p}\left(t_{0}, z_{0}\right)>\phi_{S}\left(t_{0}, z_{0}\right)$.

By the definition of $\phi_{S}$, for each $a \in \partial\left(I_{\delta} \times U_{\delta}\right)$, there exists $u_{a} \in S$ such that

$$
u_{a}(a)>\phi_{S}(a)-\frac{\delta_{2}}{2}>\tilde{p}(a)+\frac{\delta_{2}}{2} .
$$


By the continuity of $u_{a}$ and $\tilde{p}$, there exists a neighborhood $V_{a}$ of $a$ such that $u_{a}>$ $\tilde{p}-\delta_{2} / 2$ in $V_{a}$. Since $\partial\left(I_{\delta} \times U_{\delta}\right)$ is compact, there exists $a_{1}, \ldots, a_{m} \in \partial\left(I_{\delta} \times U_{\delta}\right)$ such that $\partial\left(I_{\delta} \times U_{\delta}\right)$ is covered by $\left\{V_{a_{j}}\right\}_{j=1}^{m}$. Define

$$
u=\max \left\{u_{a_{1}}, \ldots, u_{a_{m}}\right\} .
$$

Then $u \in S$ and $u>\tilde{p}+\frac{\delta_{2}}{2}$ near $\partial\left(I_{\delta} \times U_{\delta}\right)$. Define

$$
\tilde{u}(t, z)=\left\{\begin{array}{l}
u(t, z) \quad \text { if } \quad(t, z) \notin I_{\delta} \times U_{\delta}, \\
\max \{u(t, z), \tilde{p}(t, z)\} \quad \text { if } \quad(t, z) \in I_{\delta} \times U_{\delta} .
\end{array}\right.
$$

Then $\tilde{u} \in S$ and $\tilde{u}\left(t_{0}, z_{0}\right) \geq \tilde{p}\left(t_{0}, z_{0}\right)>\phi_{S}\left(t_{0}, z_{0}\right)$. We get a contradiction.

Thus $\phi_{S}$ is a supersolution to (4).

The proof is completed.

Similar to Lemma 2.12, we also have

Lemma 2.13. Assume that for every $\epsilon>0$, the problem (2) admits a continuous $\epsilon$-subbarrier which is Lipschitz in $t$ and a continuous $\epsilon$-superbarrier. Denote by $S$ the family of all continuous subsolutions to (2) which is Lipschitz in $t$. Then $\phi_{S}=\sup \{v: v \in S\}$ is a discontinuous viscosity solution to (2).

Using the comparison principle, we have the following $L^{\infty}$ a priori estimates to the viscosity solution to (2).

Proposition 2.14. Consider the Cauchy-Dirichlet problem (2) (with $\Omega$ is a smooth bounded strongly pseudoconvex domain). Suppose that either $f(t, z)>0$ for every $(t, z) \in(0, T) \times \Omega$ or $f$ is independent of $t$. If $u$ is a solution to (2) then there exists $C>0$ depending on $\Omega, \sup _{[0, T) \times \partial \Omega}|\varphi|, \min _{\bar{\Omega}} u_{0}, \sup _{[0, T) \times \Omega} f, \sup _{[0, T) \times \bar{\Omega}} F(t, z, \max \varphi)$ such that in $[0, T) \times \bar{\Omega}$.

$$
|u| \leq C \text {, }
$$

Proof. Let $\rho \in C^{2}(\bar{\Omega}) \cap P S H(\Omega)$ such that $\left.\rho\right|_{\partial \Omega}=0$ and $\left(d d^{c} \rho\right)^{n} \geq \mu(t,$.$) for all t$. We define

$$
\bar{u}=\sup _{[0, T] \times \partial \Omega} \varphi=\text { const }
$$

and

$$
\underline{u}=m+M \rho
$$

where

$$
m=\min \left\{-\sup _{[0, T) \times \partial \Omega}|\varphi|, \min _{\bar{\Omega}} u_{0}\right\}
$$

and

$$
M=\exp \left(\sup _{[0, T] \times \bar{\Omega}} \frac{F(t, z, \max \varphi)}{n}\right) .
$$

Then $\underline{u}$ is a subsolution and $\bar{u}$ is a supersolution to $(2)$. Moreover, in $\partial_{P}\left(\Omega_{T}\right)$,

$$
\underline{u} \leq u \leq \bar{u} .
$$


By the comparison principle (Theorem 2.9), we have

$$
\underline{u} \leq u \leq \bar{u}
$$

in $\Omega_{T}$.

Hence, in $[0, T) \times \bar{\Omega}$,

$$
|u| \leq C,
$$

where $C=\max \left\{\sup _{[0, T] \times \partial \Omega} \varphi,|m|+M \max _{\bar{\Omega}}(-\rho)\right\}$.

2.4. Regularizing in time. Given a bounded upper semi-continuous function $u$ : $\Omega_{T} \longrightarrow \mathbb{R}$, we consider the upper approximating sequence by Lipschitz functions in $t$

$$
u^{k}(t, x):=\sup \{u(s, x)-k|s-t|, s \in[0, T)\},(t, x) \in \Omega_{T} .
$$

If $v$ is a bounded lower semi-continuous function, we consider the lower approximating sequence of Lipschitz functions in $t$,

$$
v_{k}(t, x):=\inf \{v(s, x)+k|s-t|, s \in[0, T)\},(t, x) \in \Omega_{T} .
$$

Lemma 2.15. [EGZ15b] For $k \in \mathbb{R}^{+}, u^{k}$ is an upper semi-continuous function which satisfies the following properties:

- $u(t, z) \leq u^{k}(t, z) \leq \sup _{|s-t| \leq A / k} u(s, z)$, where $A>2$ osc $_{\Omega_{T}} u$.

- $\left|u^{k}(t, x)-u^{k}(s, x)\right| \leq k|s-t|$, for $(s, z),(t, z) \in \Omega_{T}$.

- For all $\left(t_{0}, z_{0}\right) \in[0, T-A / k] \times \Omega$, there exists $t_{0}^{*} \in[0, T)$ such that

$$
\left|t_{0}^{*}-t_{0}\right| \leq A / k \text { and } u^{k}\left(t_{0}, z_{0}\right)=u\left(t_{0}^{*}, z_{0}\right)-k\left|t_{0}-t_{0}^{*}\right| \text {. }
$$

Moreover, if u satisfies

$$
e^{\partial_{t} u+F(t, z, u)} \mu(t, z) \leq\left(d d^{c} u\right)^{n} \text { in }(0, T) \times \Omega,
$$

in the viscosity sense then the function $u^{k}$ is a subsolution of

$$
e^{\partial_{t} w+F_{k}(t, z, u)} \mu_{k}(t, z)-\left(d d^{c} w\right)^{n}=0 \text { in }(A / k, T-A / k) \times \Omega,
$$

where $F_{k}(t, z, r):=\inf _{|s-t| \leq A / k}(F(s, z, r)+k|s-t|)$ and $\mu_{k}(t, z)=\inf _{|s-t|<A / k} \mu(s, z)$. The dual statement is true for a lower semi-continuous function $v$ which is a supersolution.

2.5. Cegrell's classes. Let $\Omega$ be a bounded hyperconvex domain in $\mathbb{C}^{n}$. The following classes of plurisubharmonic functions were introduced by Cegrell [Ceg98, Ceg04]:

- $\mathcal{E}_{0}(\Omega)$ is the set of bounded psh function $u$ with $\lim _{z \rightarrow \xi} u(z)=0, \forall \xi \in \partial \Omega$ and $\int_{\Omega}\left(d d^{c} u\right)_{P}^{n}<+\infty$.

- $\mathcal{E}(\Omega)$ is the set of all $u \in P S H^{-}(\Omega)$ such that for every $z_{0} \in \Omega$, there exist a neighborhood $U$ of $z_{0}$ in $\Omega$ and a decreasing sequence $h_{j} \in \mathcal{E}_{0}(\Omega)$ such that $h_{j} \searrow u$ on $U$ and $\sup _{j} \int_{\Omega}\left(d d^{c} h_{j}\right)_{P}^{n}<\infty$.

- $\mathcal{F}(\Omega)$ is the set of all $u \in P S H^{-}(\Omega)$ such that there exists a decreasing sequence $u_{j} \in \mathcal{E}_{0}(\Omega)$ such that $u_{j} \searrow u$ on $\Omega$ and $\sup _{j} \int_{\Omega}\left(d d^{c} u_{j}\right)_{P}^{n}<+\infty$.

By [Ceg04, Blo06], $u \in \mathcal{E}(\Omega)$ iff $u$ is a non-positive psh function satisfying the following property: there exists a Borel measure $\nu$ such that, if $U \subset \Omega$ and $u_{j}$ is a sequence of bounded psh functions in $U$ satisfying $u_{j} \searrow u$ then $\left(d d^{c} u_{j}\right)_{P}^{n}$ converges weakly to $\nu$ in $U$. In this case, the Monge-Ampère operator of $u$ is defined by $\left(d d^{c} u\right)_{P}^{n}:=\nu$. 
The class $\mathcal{F}(\Omega)$ satisfies the following property: For every $u \in \mathcal{F}(\Omega)$, for each $z \in \partial \Omega$,

$$
\limsup _{\Omega \ni \xi \rightarrow z} u(\xi)=0 .
$$

Moreover, by [NP09], the comparison principle holds in the class $\mathcal{F}^{a}(\Omega)=\{u \in$ $\mathcal{F}(\Omega):\left(d d^{c} u\right)_{P}^{n}$ vanishes on all pluripolar sets $\}$.

The class $\mathcal{F}(\Omega)$ has been generalized as follows

Definition 2.16. Let $\Omega$ be a strongly pseudoconvex domain in $\mathbb{C}^{n}$. Let $\psi \in C(\partial \Omega)$. Then the class $\mathcal{F}(\Omega, \psi)$ is defined by

$$
\mathcal{F}(\Omega, \psi)=\left\{u \in P S H(\Omega): \exists v \in \mathcal{F}(\Omega) \text { such that } U_{\psi} \geq u \geq v+U_{\psi}\right\},
$$

where $U_{\psi}$ is the unique solution to the problem

$$
\left\{\begin{array}{l}
U_{\psi} \in C(\bar{\Omega} \cap P S H(\Omega)), \\
\left(d d^{c} U_{\psi}\right)_{P}^{n}=0, \\
\left.U_{\psi}\right|_{\partial \Omega}=\psi
\end{array}\right.
$$

The class $\mathcal{F}(\Omega, \psi)$ has been used to charaterize the boundary behavior in the Dirichlet problem for Monge-Ampère equation.

Theorem 2.17. [Ceg04, Aha07] Let $\Omega$ be a strongly pseudoconvex domain in $\mathbb{C}^{n}$. Let $\nu$ be a positive Borel measure in $\Omega$ and $\psi \in C(\partial \Omega)$. If $\nu(\Omega)<\infty$ and $\nu$ vanishes on all pluripolar sets then there exists a unique function $u \in \mathcal{F}(\Omega, \psi)$ such that $\left(d d^{c} u\right)_{P}^{n}=\nu$.

\section{LOCAL REGULARITY IN TIME}

In this section, we assume that $\Omega$ is a bounded domain in $\mathbb{C}^{n}$. We will prove some results on the local regularity in time of solution to (2) by using the following comparison principle.

Theorem 3.1. Let $u$ and $v$ be, respectively, a bounded subsolution and a bounded supersolution to (2).

a) Assume that for every $K \Subset \Omega$, for every $0<R<S<T$ and $\epsilon>0$, there exists $0<\delta \ll 1$ such that $(1+\epsilon) f(t+s, z) \geq f(t, z)$ for all $z \in K, 0<s<\delta$ and $R<t<S$. Then, for every $0<R<S<T$, for every $\epsilon>0$, there exists $0<\delta \ll 1$ such that

$$
u(t+s, z)<v(t, z)+\epsilon,
$$

for every $(t, z) \in(R, S) \times \Omega$ and $s \in(0, \delta)$. In particular, if either $u$ or $v$ is continuous in $t$ then $u \leq v$.

b) Assume that for every $\bar{K} \Subset \Omega$, for every $0<R<S<T$ and $\epsilon>0$, there exists $0<\delta \ll 1$ such that $(1+\epsilon) f(t, z) \geq f(t+s, z)$ for all $z \in K, 0<s<\delta$ and $R<t<S$. Then, for every $0<R<S<T$, for every $\epsilon>0$, there exists $0<\delta \ll 1$ such that

$$
u(t-s, z)<v(t, z)+\epsilon,
$$

for every $(t, z) \in(R, S) \times \Omega$ and $s \in(0, \delta)$. In particular, if either $u$ or $v$ is continuous in then $u \leq v$. 
Proof. We will prove the part a). The proof of the part b) is similar.

Let $\epsilon>0$ and $0<R<S<T$. By the semi-continuity of $u, v$ and by $u \leq v$ in $\partial_{P}\left(\Omega_{T}\right)$, there exists $\min \{R, T-S\} \gg \delta_{1}>0$ such that

$$
u(t, z) \leq v(t, z)+\epsilon,
$$

for every $(t, z) \in\left(\left[0,2 \delta_{1}\right] \times \Omega\right) \cup\left(\left[0, S+\delta_{1}\right] \times\left(\bar{\Omega} \backslash \Omega_{\delta_{1}}\right)\right)$, where

$$
\Omega_{\delta_{1}}=\left\{z \in \Omega: \operatorname{dist}(z, \partial \Omega)<\delta_{1}\right\} .
$$

By the assumption, there exists $\delta_{2} \in\left(0, \delta_{1}\right)$ such that

$$
(1+\epsilon) f\left(t_{1}, z\right) \geq f\left(t_{2}, z\right) \text { and }\left|F\left(t_{1}, z, r\right)-F\left(t_{2}, z, r\right)\right|<\epsilon
$$

for every $z \in \Omega_{\delta_{1}}, r \in[-M, M], t_{1}, t_{2} \in\left(\delta_{1}, S+\delta_{1}\right)$ with $t_{2}<t_{1}<t_{2}+\delta_{2}$. Here $M=\sup _{[0, T) \times \bar{\Omega}}|u|$.

Denote, for every $(t, z) \in[0, T) \times \bar{\Omega}$,

$$
u^{k}(t, z)=\sup \{u(s, z)-k|t-s|: s \in[0, T)\},
$$

then $u^{k}$ is Lipschitz in $t$. It follows from Lemma 2.15 and (16) that if $0<\delta<\delta_{2} / 2$ and $k>\frac{(A+1)}{\delta}$, for some $A>2 o s c_{\Omega_{T}} u$ then $u^{k}(t+\delta, z)-t \log (1+\epsilon)-(1+t) \epsilon$ is a subsolution to

$$
e^{\partial_{t} w+F(t, z, w)} \mu(t, z)=\left(d d^{c} w\right)^{n},
$$

in $\left(\delta_{1}, S\right) \times \Omega_{\delta_{1}}$. By using (15) and the comparison principle (Theorem 2.9), we get

$$
u^{k}(t+\delta, z)-t \log (1+\epsilon)-(1+t) \epsilon \leq v(t, z),
$$

for every $(t, z) \in\left(\delta_{1}, S\right) \times \Omega$.

Since $u^{k} \geq u$ and $0<\log (1+\epsilon)<\epsilon$, we have

$$
u(t+\delta, z)-(1+2 T) \epsilon \leq v(t, z),
$$

for every $(t, z) \in\left(\delta_{1}, S\right) \times \Omega_{\delta_{1}}$.

Combining (15) and (18), we obtain

$$
u(t+s, z)<v(t, z)+(1+2 T) \epsilon,
$$

for every $(t, z) \in(R, S) \times \Omega$ and $0<s<\delta$.

The proof is completed.

In Theorem 3.1, if we assume that $u$ and $v$ are continuous in $t$ then we have $u \leq v$. As a consequence, we have the following results on the Lipschitz regularity in time of viscosity solutions. This kind of regularity is necessary to define parabolic pluripotential solutions (cf. [GLZ1, GLZ2]).

Proposition 3.2. Assume that $\mu$ is non-increasing in $t$ and $u$ is a solution to (2). Suppose that there exists $C_{0}>0$ satisfying

$$
\varphi(t, z)-\varphi(s, z) \geq-C_{0}(t-s), \quad \forall z \in \partial \Omega, 0<s<t<T
$$

and for every $m>0$, there exists $C_{m}>0$ satisfying

$$
F(t, z, r)-F(s, z, r) \leq C_{m}(t-s), \quad \forall r \in[-m, m], z \in \partial \Omega, 0<s<t<T .
$$


Denote $M=\sup |u|, N=\sup |\varphi|$. Then, for every $0<B<A<T$,

$$
\frac{u(B, z)-u(A, z)}{A-B} \leq \frac{2 M}{A}+\max \left\{C_{0}, B C_{M}\right\}+n+N . B,
$$

for all $z \in \bar{\Omega}$.

In particular, $\partial_{t} u \geq-\frac{2 M}{t}-\max \left\{C_{0}, t C_{M}\right\}-n-N t$ in the viscosity sense.

Proof. The idea of the proof is similar to Theorem 4.2 in [GLZ3].

We consider $u_{A}=\frac{1}{A} u(A t, z)$ and $u_{B}=\frac{1}{B} u(B t, z)$ in $[0,1] \times \bar{\Omega}$.

By Lemma 2.5, in $(0,1) \times \Omega$, we have

$$
\left(d d^{c} u_{A}\right)^{n}=\frac{1}{A^{n}} e^{\partial_{t} u_{A}+F\left(A t, z, A u_{A}\right)} \mu(A t, z),
$$

and

$$
\left(d d^{c} u_{B}\right)^{n}=\frac{1}{B^{n}} e^{\partial_{t} u_{B}+F\left(B t, z, B u_{B}\right)} \mu(B t, z)=\frac{1}{A^{n}} e^{\partial_{t} u_{B}+F\left(B t, z, B u_{B}\right)+n \log (B / A)} \mu(B t, z),
$$

in the viscosity sense.

By the assumption, we have, for every $(t, z) \in(0,1) \times \Omega$,

$$
\mu(B t, z) \geq \mu(A t, z),
$$

and

$$
\begin{aligned}
F\left(B t, z, B u_{B}\right)+n \log (B / A) & \geq F\left(A t, z, B u_{B}\right)-C_{M}(A-B) t-\frac{n(A-B)}{B_{n}} \\
& =F\left(A t, z, A u_{B}-(A-B) u_{B}\right)-\left(C_{M}+\frac{n}{B}\right)(A-B) \\
& \geq F\left(A t, z, A u_{B}-(A-B) \sup u_{B}\right)-\left(C_{M}+\frac{n}{B}\right)(A-B) \\
& \geq F\left(A t, z, A u_{B}-N(A-B)\right)-\left(C_{M}+\frac{n}{B}\right)(A-B) .
\end{aligned}
$$

Denote

$$
\tilde{u}_{B}=u_{B}-\left(\max \left\{\frac{C_{0}}{B}, C_{M}\right\}+\frac{n}{B}\right)(A-B) t-\left(N+\frac{M}{A B}\right)(A-B) .
$$

We have, by Lemma 2.4,

$$
\left(d d^{c} \tilde{u}_{B}\right)^{n} \geq \frac{1}{A^{n}} e^{\partial_{t} \tilde{u}_{B}+F\left(A t, z, A \tilde{u}_{B}\right)} \mu(A t, z),
$$

in the viscosity sense in $(0,1) \times \Omega$. Note that $\tilde{u}_{B} \leq u_{A}$ in $\partial_{P}([0,1) \times \Omega)$. Then, by Theorem 3.1, $\tilde{u}_{B} \leq u_{A}$ in $[0,1] \times \bar{\Omega}$. In particular, for every $z \in \bar{\Omega}$,

$$
\frac{1}{A} u(A, z) \geq \frac{1}{B} u(B, z)-\left(\max \left\{\frac{C_{0}}{B}, C_{M}\right\}+\frac{n}{B}+N+\frac{M}{A B}\right)(A-B) .
$$

Hence,

$$
\frac{u(B, z)-u(A, z)}{A-B} \leq \frac{2 M}{A}+\max \left\{C_{0}, B C_{M}\right\}+n+N B,
$$

for every $z \in \bar{\Omega}$.

By the same argument, we have 
Proposition 3.3. Assume that $\mu$ is non-decreasing in $t$ and $u$ is a solution to (2). Suppose that there exists $C_{0}>0$ satisfying

$$
\varphi(t, z)-\varphi(s, z) \leq C_{0}(t-s), \quad \forall z \in \partial \Omega, 0<s<t<T,
$$

and for every $m>0$, there exists $C_{m}>0$ satisfying

$$
F(t, z, r)-F(s, z, r) \geq-C_{m}(t-s), \quad \forall r \in[-m, m], z \in \partial \Omega, 0<s<t<T .
$$

Denote $M=\sup |u|$. Then, for every $0<B<A<T$,

$$
\frac{u(A, z)-u(B, z)}{A-B} \leq \frac{2 M}{A}+\max \left\{C_{0}, B C_{M}\right\}+n+M \cdot B,
$$

for all $z \in \bar{\Omega}$.

In particular, $\partial_{t} u \leq \frac{2 M}{t}+\max \left\{C_{0}, t C_{M}\right\}+n+M t$ in the viscosity sense.

Here, the difference between the right-hand sides of (19) and (21) is due to the difference between the upper and lower estimates for $F\left(A t, z, A u_{B}-(A-B) u_{B}\right)$.

Corollary 3.4. Assume that $\mu, F, \varphi$ satisfy the conditions in Proposition 3.3. If there exists an open set $U \subset \Omega$ such that $u_{0}$ is not a maximal plurisubharmonic function in $U$ and $\lim _{t \rightarrow 0^{+}} t \log \sup _{z \in U} f(t, z)=-\infty$ then (2) does not admit a solution.

Combining Proposition 3.2 and Proposition 3.3, we have

Corollary 3.5. Assume that $\mu$ is independent of $t$ and $u$ is a solution to (2). Suppose that there exists $C_{0}>0$ satisfying

$$
|\varphi(t, z)-\varphi(s, z)| \leq C_{0}|t-s|, \quad \forall z \in \partial \Omega, s, t \in[0, T),
$$

and for every $m>0$, there exists $C_{m}>0$ satisfying

$$
|F(t, z, r)-F(s, z, r)| \leq C_{m}|t-s|, \quad \forall r \in[-m, m], z \in \partial \Omega, s, t \in[0, T) .
$$

Denote $M=\sup |u|$. Then, for every $0<B<A<T$,

for all $z \in \bar{\Omega}$.

$$
\frac{|u(A, z)-u(B, z)|}{A-B} \leq \frac{2 M}{A}+\max \left\{C_{0}, B C_{M}\right\}+n+M \cdot B,
$$

In particular, $\left|\partial_{t} u\right| \leq \frac{2 M}{t}+\max \left\{C_{0}, t C_{M}\right\}+n+M t$ in the viscosity sense.

We then have the following corollary.

Corollary 3.6. Assume that $\mu, F$ and $\varphi$ are independent of $t$. If (2) admits a viscosity solution then for every $0<t<T$, there exists $C_{t}>0$ such that $\left(d d^{c} u(t, z)\right)^{n} \leq C_{t} \mu(z)$ in the viscosity sense in $\Omega$. In particular, $\left(u_{0}, \mu\right)$ is admissible.

\section{The EXISTEnCE OF SOLUtion}

In this section, we prove Theorem 1.2 and Theorem 1.3. Assume that $\Omega \subset \mathbb{C}^{n}$ is a smooth bounded strongly pseudoconvex domain. Consider the Cauchy-Dirichlet problem spelt out in the Introduction

$$
\left\{\begin{array}{l}
e^{\partial_{t} u+F(t, z, u)} \mu(t, z)=\left(d d^{c} u\right)^{n} \quad \text { in } \quad \Omega_{T}, \\
u=\varphi \quad \text { in } \quad[0, T) \times \partial \Omega, \\
u(0, z)=u_{0}(z) \quad \text { in } \bar{\Omega} .
\end{array}\right.
$$


4.1. The construction of $\epsilon$-subbarriers and $\epsilon$-superbarriers. The $\epsilon$-subbarriers and $\epsilon$-superbarriers are important for proving the existence of solutions to the problem (22). In [EGZ15b], Eyssidieux-Guedj-Zeriahi have given a method to construct $\epsilon$-subbarriers and $\epsilon$-superbarriers in the case where $\phi$ and $f$ are independent of $t$ (see [EGZ15b, Proposition 5.9]). In this section, we introduce several results about the construction of $\epsilon$-subbarriers and $\epsilon$-superbarriers in the general case.

Proposition 4.1. For all $\epsilon>0$, there exists a continuous $\epsilon$-subbarrier for (2) which is Lipschitz in $t$.

Proof. Let $\rho \in C^{2}(\bar{\Omega}) \cap P S H(\Omega)$ such that $\left.\rho\right|_{\partial \Omega}=0,\left.\nabla \rho\right|_{\partial \Omega} \neq 0$ and $d d^{c} \rho \geq d d^{c}|z|^{2}$. Denote $c=\sup _{\Omega}(-\rho)$. Then, there exists $M_{1} \gg 1$ such that the function

$$
\underline{u}_{1}=u_{0}+\frac{\epsilon(\rho-c)}{2 c}-M_{1} t
$$

is a subsolution to $(22)$ satisfying $\underline{u}_{1} \leq \varphi$ in $[0, T] \times \partial \Omega$.

Let $\varphi_{\epsilon} \in C^{\infty}\left(\mathbb{R} \times \mathbb{C}^{n}\right)$ such that

$$
\varphi-\frac{\epsilon}{2} \leq \varphi_{\epsilon} \leq \varphi
$$

in $[0, T] \times \partial \Omega$. Then, there exists $M_{2} \gg 1$ such that the function

$$
\underline{u}_{2}=\varphi_{\epsilon}-\frac{\epsilon}{2}+M_{2} \rho,
$$

is a subsolution to (22) satisfying $\underline{u}_{2} \leq u_{0}$ in $\{0\} \times \bar{\Omega}$.

Now, we define $\underline{u}=\max \left\{\underline{u}_{1}, \underline{u}_{2}\right\}$. It is clear that $\underline{u}$ is a continuous $\epsilon$-subbarrier for $(22)$.

Lemma 4.2. 1) Let $0<\epsilon_{0}<T$. Let $u$ be a bounded continuous subsolution to $(22)$ in $\left[0, \epsilon_{0}\right) \times \bar{\Omega}$. Then, for every $0<\epsilon<\epsilon_{0}$, there exists a continuous subsolution $\tilde{u}$ to $(22)$ on $[0, T) \times \Omega$ such that

$$
\left\{\begin{array}{l}
\tilde{u} \leq u \text { in }\left[0, \epsilon_{0}\right) \times \bar{\Omega} \\
\tilde{u}=u \text { in }[0, \epsilon) \times \bar{\Omega} .
\end{array}\right.
$$

Moreover, if $u$ is Lipschitz in $t$ then $\tilde{u}$ is also Lipschitz in $t$.

2) Let $0<\epsilon_{0}<T$. Let $v$ be a bounded continuous supersolution to (22) in $\left[0, \epsilon_{0}\right) \times \bar{\Omega}$. Then, for every $0<\epsilon<\epsilon_{0}$, there exists a continuous supersolution $\tilde{v}$ to $(22)$ on $[0, T) \times \Omega$ such that

$$
\left\{\begin{array}{l}
\tilde{v} \geq v \text { in }\left[0, \epsilon_{0}\right) \times \bar{\Omega} \\
\tilde{v}=v \text { in }[0, \epsilon) \times \bar{\Omega}
\end{array}\right.
$$

Moreover, if $v$ is Lipschitz in $t$ then $\tilde{v}$ is also Lipschitz in $t$.

Proof. 1) Denote

$$
M=\sup _{\left[0, \epsilon_{0}\right) \times \bar{\Omega}} u, m=\min \left\{\inf _{\left[0, \epsilon_{0}\right) \times \bar{\Omega}} u(t, z), \min _{[0, T] \times \partial \Omega} \varphi(t, z)\right\},
$$

and

$$
M_{F}=\max _{[0, T] \times \bar{\Omega}} F(t, z, m), M_{f}=\sup _{[0, T] \times \Omega} f(t, z) .
$$

Let $\rho \in C^{2}(\bar{\Omega}) \cap P S H^{-}(\Omega)$ such that $\left(d d^{c} \rho\right)^{n} \geq e^{M_{F}} M_{f} d V$. We define 


$$
h(t)=\left\{\begin{array}{l}
0 \quad \text { for } t<\epsilon, \\
C(t-\epsilon) \quad \text { for } t \geq \epsilon,
\end{array}\right.
$$

where $C=1+\frac{M-m+\max (-\rho)}{\epsilon_{0}-\epsilon}$.

Then

$$
\tilde{u}=\left\{\begin{array}{l}
\max \{u(t, z)-h(t), m+\rho\} \\
m+\rho \quad \text { in } \quad\left[\epsilon_{0}, T\right) \times \bar{\Omega},
\end{array} \quad \text { in } \quad\left[0, \epsilon_{0}\right) \times \bar{\Omega},\right.
$$

is a continuous subsolution to (2) satisfying

$$
\left\{\begin{array}{l}
\tilde{u} \leq u \text { in }\left[0, \epsilon_{0}\right) \times \bar{\Omega} \\
\tilde{u}=u \text { in }[0, \epsilon) \times \bar{\Omega}
\end{array}\right.
$$

2) Denote

$$
m=\inf _{\left[0, \epsilon_{0}\right) \times \bar{\Omega}} v \text { and } M=\max \left\{\sup _{\left[0, \epsilon_{0}\right) \times \bar{\Omega}} v, \max _{[0, T] \times \partial \Omega} \varphi\right\}
$$

We define

$$
h(t)=\left\{\begin{array}{l}
0 \quad \text { for } t<\epsilon, \\
C(t-\epsilon) \quad \text { for } t \geq \epsilon
\end{array}\right.
$$

where $C=1+\frac{M-m}{\epsilon_{0}-\epsilon}$.

Then

$$
\tilde{v}=\left\{\begin{array}{l}
\min \{v(t, z)+h(t), M\} \\
M \quad \text { in }\left[\epsilon_{0}, T\right) \times \bar{\Omega},
\end{array} \quad \text { in } \quad\left[0, \epsilon_{0}\right) \times \bar{\Omega},\right.
$$

is a continuous supersolution to (2) satisfying

$$
\left\{\begin{array}{l}
\tilde{v} \geq v \text { in }\left[0, \epsilon_{0}\right) \times \bar{\Omega} \\
\tilde{v}=v \text { in }[0, \epsilon) \times \bar{\Omega}
\end{array}\right.
$$

Proposition 4.3. If $\left(u_{0}(z), \mu(0, z)\right)$ is admissible then for all $\epsilon>0$, there exists a continuous $\epsilon$-superbarrier for (22) which is Lipschitz in $t$.

In order to prove Proposition 4.3, we need the following lemma

Lemma 4.4. For every $p>1$, there exists a continuous function $A_{p}:(0, \infty) \rightarrow$ $(0, \infty)$ with $\lim _{t \rightarrow 0^{+}} A_{p}(t)=0$ such that: for every $g \in L^{p}(\Omega)$, the unique solution $u$ to the problem

$$
\left\{\begin{array}{l}
u \in C(\bar{\Omega}) \cap P S H(\Omega) \\
\left(d d^{c} u\right)^{n}=g d V \\
\left.u\right|_{\partial \Omega}=0
\end{array}\right.
$$

satisfies $\sup _{\Omega}|u| \leq A_{p}\left(\|g\|_{L^{p}(\Omega)}\right)$.

Lemma 4.4 is an immediate corollary of the following theorem 
Theorem 4.5. [GKZ08, Theorem 1.1] Fix $0 \leq g \in L^{p}(\Omega), p>1$. Let $\psi_{1}, \psi_{2}$ be two bounded psh functions in $\Omega$ such that $\left(d d^{c} \psi_{1}\right)^{n}=g d V$, and $\lim _{\inf } \operatorname{sa\Omega }_{2}\left(\psi_{1}-\psi_{2}\right)(z) \geq$ 0 . Fix $r \geq 1$ and $0 \leq \gamma<r /[n q+r], 1 / p+1 / q=1$. Then

$$
\sup \left(\psi_{2}-\psi_{1}\right) \leq C\left\|\max \left\{\psi_{2}-\psi_{1}, 0\right\}\right\|_{L^{r}(\Omega)}^{\gamma},
$$

for some uniform constant $C=C\left(\gamma,\|g\|_{L^{p}(\Omega)}\right)$. Moreover, for each $0 \leq \gamma<r /[n q+$ $r]$,

$$
\lim _{\|g\|_{L^{p}(\Omega)} \rightarrow 0^{+}} C\left(\gamma,\|g\|_{L^{p}(\Omega)}\right)=0 .
$$

The last assertion of Theorem 4.5 is not mentioned in [GKZ08, Theorem 1.1] but it is deduced from the proof of this theorem (see [GKZ08, pages 1071-1073]).

Proof of Proposition 4.3. Since $\left(u_{0}(z), \mu(0, z)\right)$ is admissible, there exist $u_{\epsilon} \in C(\bar{\Omega})$ and $C_{\epsilon}>0$ such that $u_{0}+\frac{\epsilon}{4} \leq u_{\epsilon} \leq u_{0}+\frac{\epsilon}{2}$ and $\left(d d^{c} u_{\epsilon}\right)^{n} \leq e^{C_{\epsilon}} \mu(0, z)$ in the viscosity sense. Denote

$$
M_{1}=\sup \left\{\left|F\left(t, z, u_{0}(z)\right)\right|:(t, z) \in(0, T) \times \Omega\right\} .
$$

By using the definition, we have $\hat{u}(t, z):=u_{\epsilon}(z)+\left(C_{\epsilon}+M_{1}\right) t$ is a viscosity supersolution to the equation

$$
\left(d d^{c} w\right)^{n}=e^{\partial_{t} w+F(t, z, w)} \mu(0, z)
$$

in $(0, T) \times \Omega$.

For every $0<\delta<T$, we denote

$$
f_{\delta}(z)=\sup \{|f(t, z)-f(0, z)|: 0 \leq t \leq \delta\} .
$$

Then $f_{\delta} \in C(\Omega) \cap L^{\infty}(\Omega)$ (by Lemma 2.8) and $f_{\delta} \searrow 0$ as $\delta \searrow 0$. Hence

$$
\lim _{\delta \rightarrow 0^{+}}\left\|f_{\delta}\right\|_{L^{2}(\Omega)}=0 \text {. }
$$

Therefore, we can choose $0<\delta_{1}<T$ such that $A_{2}\left(e^{C_{\epsilon}+M_{1}+M_{2}}\left\|f_{\delta_{1}}\right\|_{L^{2}(\Omega)}\right)<\epsilon / 2$, where $A_{2}$ is defined as in Lemma 4.4 and

$$
M_{2}:=\sup \left\{F\left(t, z, \sup _{[0, T] \times \partial \Omega} \varphi\right):(t, z) \in[0, T] \times \bar{\Omega}\right\} .
$$

Define by $\rho$ the unique solution to the problem

$$
\left\{\begin{array}{l}
\rho \in P S H(\Omega) \cap C(\bar{\Omega}), \\
\left(d d^{c} \rho\right)^{n}=e^{C_{\epsilon}+M_{1}+M_{2}} f_{\delta_{1}} d V, \\
\left.\rho\right|_{\partial \Omega}=0 .
\end{array}\right.
$$

Then, by using Lemma 2.7, we have $\hat{u}-\rho$ is a supersolution to the equation

$$
\left(d d^{c} w\right)^{n}=e^{\partial_{t} w+F(t, z, w)} \mu(t, z),
$$

in $\left(0, \delta_{1}\right) \times \Omega$. Moreover, by Theorem 4.5, we have $0 \leq-\rho \leq \epsilon / 2$.

Choose $0 \leq \delta_{2} \leq \delta_{1}$ such that $\hat{u}(t, z) \geq \varphi(t, z)$ for every $(t, z) \in\left(0, \delta_{2}\right) \times \partial \Omega$. Then $\hat{u}-\rho$ is a supersolution to $(22)$ in $\left[0, \delta_{2}\right) \times \bar{\Omega}$ which is Lipschitz in $t$. Moreover $u_{0}(z) \leq$ $\hat{u}(0, z)-\rho(z) \leq u_{0}(z)+\epsilon$ in $\Omega$. By using Lemma 4.2, there exists a supersolution $\underline{u}_{1}$ to $(22)$ in $[0, T) \times \bar{\Omega}$ which is Lipschitz in $t$ and satisfies $u_{0}(z) \leq \underline{u}_{1}(0, z) \leq u_{0}(z)+\epsilon$ in $\Omega$.

Let $\varphi^{\epsilon} \in C^{\infty}\left(\mathbb{R} \times \mathbb{C}^{n}\right)$ such that

$$
\varphi \leq \varphi^{\epsilon} \leq \varphi+\epsilon
$$


in $[0, T] \times \partial \Omega$. For every $t \in[0, T]$, we denote by $\bar{u}_{2}(t, z)$ the unique solution to the equation

$$
\left\{\begin{array}{l}
\bar{u}_{2}(t, \cdot) \in P S H(\Omega) \cap C(\bar{\Omega}) \\
\left(d d^{c} \bar{u}_{2}(t, z)\right)_{P}^{n}=0 \\
\left.\bar{u}_{2}(t, z)\right|_{\partial \Omega}=\left.\varphi^{\epsilon}(t, z)\right|_{\partial \Omega}
\end{array}\right.
$$

By [BT76, Theorem A] we have

$$
\left|\bar{u}_{2}\left(t_{1}, z\right)-\bar{u}_{2}\left(t_{2}, z\right)\right| \leq \sup \left\{\left|\varphi^{\epsilon}(t, \xi)-\varphi^{\epsilon}(t, \xi)\right|: \xi \in \partial \Omega\right\},
$$

for every $t_{1}, t_{2} \in[0, T]$, for all $z \in \Omega$.

Then $\bar{u}_{2}:[0, T] \times \bar{\Omega} \rightarrow \mathbb{R}$ is Lipschitz in $t$. In particular, $\bar{u}_{2} \in C([0, T] \times \bar{\Omega})$. It is easy to see that $\bar{u}_{2}$ is a supersolution to (22).

Now, we define $\bar{u}=\min \left\{\bar{u}_{1}, \bar{u}_{2}\right\}$. It is clear that $\bar{u}$ is a continuous $\epsilon$-superbarrier for $(22)$.

Remark 4.6. The converse statement of Proposition 4.3 is false. For example, if $\Omega$ is the unit ball, $u_{0}=|z|^{2}-1, \varphi=0, F=0, \mu=t d V$ then for every $T>0$,

$$
\bar{u}_{T}(t, z)=\min \left\{0, u_{0}-t \log t+e^{T} t\right\},
$$

is an $\epsilon$-superbarrier for $(22)$ in $[0, T) \times \bar{\Omega}$ for every $\epsilon>0$. But $\left(u_{0}, \mu(0, z)\right)=$ $\left(|z|^{2}-1,0\right)$ is not admissible.

Proposition 4.7. Let $\epsilon>0$. If there exists a continuous $\epsilon$-superbarrier $u$ for $(2)$ in $[0, S) \times \bar{\Omega}$ for some $0<S<T$, then there exists a continuous $\epsilon$-superbarrier $\tilde{u}$ for $(2)$ in $[0, T) \times \bar{\Omega}$. Moreover, if $u$ is Lipschitz in $t$ then $\tilde{u}$ is also Lipschitz in $t$.

Proof. By the assumption and by Lemma 4.2, there exists a continuous supersolution $u_{1}$ to $(22)$ in $[0, T) \times \bar{\Omega}$ such that $u_{0}(z) \leq u_{1}(0, z) \leq u_{0}(z)+\epsilon$ for all $z \in \Omega$.

Let $\varphi^{\epsilon} \in C^{\infty}\left(\mathbb{R} \times \mathbb{C}^{n}\right)$ such that

$$
\varphi \leq \varphi^{\epsilon} \leq \varphi+\epsilon
$$

in $[0, T] \times \partial \Omega$.

Let $u_{2} \in C([0, T] \times \bar{\Omega})$ such that $u_{2}=\varphi^{\epsilon}$ in $[0, T] \times \partial \Omega$ and $u_{2}(t,$.$) is maximal$ plurisubharmonic in $\Omega$ for every $t \in[0, T]$.

Then $\tilde{u}=\min \left\{u_{1}, u_{2}\right\}$ is a continuous $\epsilon$-superbarrier for $(22)$ in $[0, T) \times \bar{\Omega}$.

For $j=1,2$, we assume that

- $F_{j}(t, z, r)$ is continuous in $[0, T] \times \bar{\Omega} \times \mathbb{R}$ and non-decreasing in $r$.

- $\varphi_{j}(t, z)$ is a continuous function in $[0, T] \times \partial \Omega$ such that $u_{0}(z)=\varphi_{j}(0, z)$ in $\partial \Omega$.

We consider the following problems

$$
\left\{\begin{array}{l}
e^{\partial_{t} u+F_{1}(t, z, u)} \mu(t, z)=\left(d d^{c} u\right)^{n} \quad \text { in } \quad(0, T) \times \Omega, \\
u=\varphi_{1} \quad \text { in } \quad[0, T) \times \partial \Omega, \\
u(0, z)=u_{0}(z) \quad \text { in } \bar{\Omega},
\end{array}\right.
$$

and

$$
\left\{\begin{array}{l}
e^{\partial_{t} u+F_{2}(t, z, u)} \mu(t, z)=\left(d d^{c} u\right)^{n} \quad \text { in } \quad(0, T) \times \Omega \\
u=\varphi_{2} \quad \text { in } \quad[0, T) \times \partial \Omega, \\
u(0, z)=u_{0}(z) \quad \text { in } \bar{\Omega} .
\end{array}\right.
$$


Proposition 4.8. Let $\epsilon_{0}>0$. If there exists a continuous $\epsilon_{0}$-superbarrier $\bar{u}_{1}$ for the problem (28) then for every $\epsilon>\epsilon_{0}$, there exists a continuous $\epsilon$-superbarrier $\bar{u}_{2}$ for the problem (29). Moreover, if $\bar{u}_{1}$ is Lipschitz in $t$ then $\bar{u}_{2}$ is Lipschitz in $t$.

Proof. Since $u_{0}(z)=\varphi_{1}(0, z)=\varphi_{2}(0, z)$ for every $z \in \partial \Omega$, there exists $\delta>0$ such that

$$
\left|\varphi_{1}(t, z)-\varphi_{2}(t, z)\right|<\frac{\epsilon-\epsilon_{0}}{3}
$$

for every $(t, z) \in[0, \delta] \times \partial \Omega$. Let $C>0$ such that

$$
e^{\partial_{t}\left(\bar{u}_{1}+C t\right)+F_{2}\left(t, z, \bar{u}_{1}+C t\right)} \mu(t, z) \geq\left(d d^{c}\left(\bar{u}_{1}+C t\right)\right)^{n},
$$

in the viscosity sense in $[0, \delta) \times \partial \Omega$.

Let $\delta_{0}=\min \left\{\delta, \frac{\epsilon-\epsilon_{0}}{3 C}\right\}$. Then $\bar{u}_{1}+C t+\frac{\epsilon-\epsilon_{0}}{3}$ is a continuous $\epsilon$-superbarrier for the problem $(29)$ in $\left[0, \delta_{0}\right) \times \partial \Omega$. Hence, it follows from Proposition 4.7 that there exists a continuous $\epsilon$-superbarrier $\bar{u}_{2}$ for the problem (29).

4.2. The existence of solution. Now we prove some results about the existence of solution to (2). Theorem 1.2 is an immediate corollary of the following:

Theorem 4.9. Suppose that for every $\epsilon>0$, there exists a continuous $\epsilon$-superbarrier for (2). Assume that for every $K \Subset \Omega$, for every $0<R<S<T$ and $\epsilon>0$, there exists $0<\delta \ll 1$ such that

$$
(1+\epsilon) f(t+s, z) \geq f(t, z)
$$

and

$$
(1+\epsilon) f(t, z) \geq f(t+s, z)
$$

for all $z \in K, 0<s<\delta$ and $R<t<S$.

Then (2) admits a unique solution $u$. Moreover, $(u(t, z), \mu(t, z))$ is admissible for every $0<t<T$. If (30) holds in the case $S=T$ then $u$ can be extended continuously to $[0, T] \times \bar{\Omega}$ and $(u(T, z), \mu(T, z))$ is admissible.

Proof. Denote by $u$ the supremum of continuous subsolutions to (2). Then, by Proposition 4.1 and Lemma 2.12, $u=u_{*}$ is a supersolution to (2) and $u^{*}$ is a subsolution to (2).

By Theorem 3.1, for every $(t, z) \in(0, T) \times \Omega$, for each $\epsilon>0$, there exists $\delta>0$ such that

$$
u(t, z)+\epsilon \geq u^{*}(s, z) \geq u(s, z),
$$

for all $0<|s-t|<\delta$. Hence, by using the fact that $u$ is lower semi-continuous, we get that $u$ is continuous in $t$.

Then, by Theorem 3.1, for every $\epsilon>0$ and $(t, z) \in(0, T) \times \Omega$,

$$
u^{*}(t, z) \leq \lim _{s \rightarrow t} u(s, z)+\epsilon=u(t, z)+\epsilon .
$$

Letting $\epsilon \rightarrow 0$, we obtain $u^{*}=u$.

Thus $u$ is a solution to (2). The uniqueness of solution holds due to Theorem 3.1.

Now, we consider the case where (30) holds in the case $S=T$. For every $t>T$, we define

$$
\mu(t, .)=\mu(T, .), F(t, . . .)=F(T, ., .) \text { and } \varphi(t, .)=\varphi(T, .) .
$$


Denote by $\tilde{u}$ the supremum of continuous subsolutions to the problem

$$
\left\{\begin{array}{l}
e^{\partial_{t} u+F(t, z, u)} \mu(t, z)=\left(d d^{c} u\right)^{n} \quad \text { in } \quad(0, \infty) \times \Omega, \\
u=\varphi \quad \text { in } \quad[0, \infty) \times \partial \Omega, \\
u(0, z)=u_{0}(z) \quad \text { in } \bar{\Omega},
\end{array}\right.
$$

Then, it follows from Lemma 4.2 that $\tilde{u}=u$ in $[0, T) \times \bar{\Omega}$. By the continuity of $u$, we also have $\tilde{u}^{*}=u$ in $[0, T) \times \bar{\Omega}$.

By Lemma 2.12, $\tilde{u}^{*}$ is a supersolution to (32). Applying the part a) of Theorem 3.1 , for every $\epsilon>0$, there exists $\delta_{1}>0$ such that

$$
u(t, z)=\tilde{u}^{*}(t, z)<\tilde{u}(s, z)+\epsilon=u(s, z)+\epsilon,
$$

for every $T-\delta_{1}<s<t<T$ and $z \in \Omega$. Choose $\rho \in C^{2}(\bar{\Omega}) \cap P S H(\Omega)$ such that $-1 \leq \rho \leq 0$ and $d d^{c} \rho>0$. Choose $C \gg 1$ such that

$$
\left(d d^{c} \rho\right)^{n} \geq e^{-C+\sup F(., ., \sup \varphi)} \sup f d V .
$$

Here, we recall that $\mu(t, z)=f(t, z) d V$.

Then, for every $1>\epsilon>0$, there exists $0<\delta_{2}<\delta_{1}$ such that, for every $s \in$ $\left(T-\delta_{2}, T\right)$, the function

$$
u(s, z)+\frac{\epsilon}{3} \rho(z)-\left(C+n \log \frac{2}{\epsilon}\right)(t-s)-\frac{\epsilon}{3},
$$

is a subsolution to

$$
e^{\partial_{t} w+F(t, z, w)} \mu(t, z)=\left(d d^{c} w\right)^{n}
$$

in $(s, T) \times \Omega$.

Choose $\delta_{2} \ll 1$ such that $|\varphi(t, z)-\varphi(s, z)|<\epsilon / 3$ for every $z \in \partial \Omega$ and $t, s \in$ $\left[T-\delta_{2}, T\right]$. Using Theorem 2.9, for every $T-\delta_{2}<s<t<T$ and $z \in \Omega$,

$$
u(s, z)+\frac{\epsilon}{3} \rho(z)-\left(C+n \log \frac{2}{\epsilon}\right) t-\frac{\epsilon}{3}<u(t, z) .
$$

Choose $0<\delta_{3}<\delta_{2}$ such that $\left(C+n \log \frac{2}{\epsilon}\right) \delta_{3}<\frac{\epsilon}{3}$. We have

$$
u(s, z)-\epsilon<u(t, z),
$$

for every $T-\delta_{3}<s<t<T$ and $z \in \Omega$.

Combining (33) and (36), we get that $u(t, z)$ converges uniformly to a continuous plurisubharmonic function $u(T, z)$ as $t \nearrow T$. By using the condition (30) and applying Lemma 2.15 , we obtain that $(u(T, z), \mu(T, z))$ is admissible.

Corollary 4.10. If there exist $0 \leq f_{0}, f_{1} \in C(\Omega)$ such that $\left(u_{0}, f_{0} d V\right)$ is admissible and $f(t, z)=t f_{1}(z)+(T-t) f_{0}(z)$ then (2) has a unique solution.

Remark 4.11. By combining Theorem 4.9 and Lemma 2.15, if $\mu$ is independent of then (2) admits a solution iff $\left(u_{0}, \mu\right)$ is admissible. Hence, by Corollary 3.6, Definition 1.1 is equivalent to the definition of the admissibility in [EGZ15b].

Theorem 4.12. Let $u \in C^{0}([0, T] \times \bar{\Omega})$ such that

$$
e^{\partial_{t} u+F(t, z, u)} \mu(t, z)=\left(d d^{c} u\right)^{n},
$$

in $((0, S) \cup(S, T)) \times \Omega$ in the viscosity sense for some $S \in(0, T)$.

If $(u(S, z), \mu(S, z))$ is admissible then $u$ satisfies (37) in the viscosity sense in $(0, T) \times \Omega$. 
Proof. By Lemma 2.6, it remains to show that, for every $\epsilon>0$, there exist a subsolution $\underline{u}_{\epsilon}$ and a supersolution $\bar{u}_{\epsilon}$ to $(37)$ in $(0, T) \times \Omega$ such that

$$
\left|u(t, z)-\underline{u}_{\epsilon}(t, z)\right|<\epsilon \text { and }\left|u(t, z)-\bar{u}_{\epsilon}(t, z)\right|<\epsilon,
$$

for every $(t, z) \in(0, T) \times \Omega$. that

Let $\rho \in C^{2}(\bar{\Omega}) \cap P S H(\Omega)$ such that $-1 \leq \rho \leq 0$ and $d d^{c} \rho>0$. Let $C_{1} \gg 1$ such

$$
\left(d d^{c} \rho\right)^{n} \geq e^{-C_{1}+\sup F(., ., \sup u)} \sup f d V .
$$

Here, we recall that $\mu(t, z)=f(t, z) d V$.

Denote, for every $0 \leq t \leq S$,

$$
h(t)=\sup \{|u(S-s, z)-u(S, z)|: z \in \Omega, s \in[0, t]\} .
$$

Then $h$ is a non-decreasing function with $h(0)=0$. Moreover, by Lemma 2.8, $h$ is continuous.

Let $1>\epsilon>0$. Then there exists $\delta_{1} \in(0, S)$ such that

$$
\max \left\{h\left(\delta_{1}\right),\left(C_{1}+n \log \frac{3}{\epsilon}\right) \delta_{1}\right\}<\frac{\epsilon}{3} .
$$

For $(t, z) \in(0, S) \times \bar{\Omega}$, we denote

$$
\tilde{h}(t, z)=h(S-t)-\left(C_{1}+n \log \frac{3}{\epsilon}\right)\left(t-S+\delta_{1}\right)+\frac{\epsilon\left(t-S+\delta_{1}\right)}{3 \delta_{1}} \rho(z) .
$$

For every $(t, z) \in[0, T) \times \bar{\Omega}$, we define

$$
h_{\epsilon}(t, z)=\left\{\begin{array}{cc}
h\left(\delta_{1}\right) & \text { if } t \in\left[0, S-\delta_{1}\right], \\
\tilde{h}(t, z) & \text { if } t \in\left[S-\delta_{1}, S\right], \\
\frac{\epsilon \rho(z)}{3}-\left(C_{1}+n \log \frac{3}{\epsilon}\right) \delta_{1} & \text { if } t \in[S, T),
\end{array}\right.
$$

and

$$
\underline{u}_{\epsilon}(t, z)=u(t, z)+h_{\epsilon}(t, z)-\frac{\epsilon}{3} .
$$

Then $\left|u(t, z)-\underline{u}_{\epsilon}(t, z)\right|<\epsilon$ for every $(t, z) \in(0, T) \times \Omega$ and we will show that $\underline{u}_{\epsilon}$ is a subsolution to $(37)$ in $(0, T) \times \Omega$.

By the assumption and by the fact that $h_{\epsilon}(t, z)$ is non-increasing in $t$ and plurisubharmonic in $z$, we have $\underline{u}_{\epsilon}$ is a subsolution to $(37)$ in $((0, S) \cup(S, T)) \times \Omega$. It remains to show that, for every $z_{0} \in \Omega$, for every upper test function $p$ for $\underline{u}_{\epsilon}$ at $\left(S, z_{0}\right)$,

$$
\left(d d^{c} p\left(S, z_{0}\right)\right)^{n} \geq e^{\partial_{t} p\left(S, z_{0}\right)+F\left(S, z_{0}, p\left(S, z_{0}\right)\right)} \mu\left(S, z_{0}\right) .
$$

Note that, by the definition of $h(t)$, we have $u(S-t, z)+h(t) \geq u(S, z)$, for every $(t, z) \in\left(0, \delta_{1}\right) \times \Omega$. Then,

$$
\underline{u}_{\epsilon}(S-t, z) \geq \underline{u}_{\epsilon}(S, z)+\left(C_{1}+n \log \frac{3}{\epsilon}\right) t-t \rho(z) \geq \underline{u}_{\epsilon}(S, z)+\left(C_{1}+n \log \frac{3}{\epsilon}\right) t,
$$

for every $(t, z) \in\left(0, \delta_{1}\right) \times \Omega$. Hence, for every $z_{0} \in \Omega$, for every upper test function $p$ for $\underline{u}_{\epsilon}$ at $\left(S, z_{0}\right)$, we have

$$
\partial_{t} p\left(S, z_{0}\right) \leq-\left(C_{1}+n \log \frac{3}{\epsilon}\right) .
$$


By [EGZ15b, Corollary 3.7], $u(t, z)$ is plurisubharmonic in $z$ for every $t \in(0, S)$. Moreover, since $u$ is continuous on the compact set $[0, S] \times \bar{\Omega}$, we have $u(t, z)$ converges uniformly to $u(S, z)$ as $t \nearrow S$. Then $u(S, z)=\lim _{t \rightarrow S^{-}} u(t, z)$ is plurisubharmonic. Since $p(S, z)-h_{\epsilon}(S, z)+\frac{\epsilon}{3}$ is a upper test function for $u(S, z)$ at $z_{0}$, we have, by [EGZ11, Proposition 1.3],

$$
d d^{c}\left(p(S, z)-h_{\epsilon}(S, z)\right) \geq 0
$$

at $z_{0}$. Hence, we have, at $\left(S, z_{0}\right)$

$$
\left(d d^{c} p\right)^{n} \geq\left(d d^{c} h_{\epsilon}\right)^{n}=\frac{\epsilon^{n}}{3^{n}}\left(d d^{c} \rho\right)^{n} \geq \frac{\epsilon^{n}}{3^{n}}\left(d d^{c} \rho\right)^{n} e^{-C_{1}+\sup F(., ., \sup u)} \sup f d V .
$$

Combining (39) and (40), we get (38). Thus, $\underline{u}_{\epsilon}$ is a subsolution to $(37)$ in $(0, T) \times \Omega$.

Now, we construct $\bar{u}_{\epsilon}$. Since $(u(S, z), \mu(S, z))$ is admissible, there exist $C_{2}>0$ and $u^{\epsilon} \in C(\bar{\Omega}) \cap P S H(\Omega)$ such that $u(S, z)+\frac{\epsilon}{3}<u^{\epsilon}(z)<u(S, z)+\frac{2 \epsilon}{3}$ and $\left(d d^{c} u^{\epsilon}\right)^{n} \leq$ $e^{C_{2}+\inf F(.,, \inf u)} \mu(S, z)$.

Let $\delta_{2}>0$ such that $C_{2} \delta_{2}<\frac{\epsilon}{3}$ and $\frac{\epsilon}{3}<u(t, z)-u^{\epsilon}(z)<\frac{2 \epsilon}{3}$ for every $(t, z) \epsilon$ $\left(S-\delta_{2}, S\right) \times \Omega$. For every $(t, z) \in[0, T) \times \bar{\Omega}$, we define the function $\bar{u}_{\epsilon}$ by

$$
\begin{cases}u(t, z) & \text { if } t \in\left[0, S-\delta_{2}\right], \\ \min \left\{u(t, z)+\frac{\epsilon}{\delta_{2}}\left(t-S+\delta_{2}\right), u^{\epsilon}(t, z)+C_{2}\left(t-S+\delta_{2}\right)+\frac{2 \epsilon}{3}\right\} & \text { if } t \in\left[S-\delta_{2}, S\right], \\ \min \left\{u^{\epsilon}(t, z)+C_{2}\left(t-S+\delta_{2}\right)+\frac{2 \epsilon}{3}, u(t, z)+\epsilon\right\} & \text { if } t \in[S, T) .\end{cases}
$$

Then $\bar{u}_{\epsilon}$ is a supersolution to (37) in $(0, T) \times \Omega$ satisfying $\left|u(t, z)-\underline{u}_{\epsilon}(t, z)\right|<\epsilon$ for every $(t, z) \in(0, T) \times \Omega$. The proof is completed.

By using Theorem 4.9 and Theorem 4.12, we have the following:

Theorem 4.13. Assume that there exist $t_{1}, \ldots, t_{m}$ with $0=t_{0}<t_{1}<\ldots<t_{m}=T$ satisfying

i) For every $K \Subset \Omega$, for every $\epsilon>0$ and $t_{k-1}<R<t_{k}(1 \leq k \leq m)$, there exists $0<\delta \ll 1$ such that

$$
(1+\epsilon) f(t+s, z) \geq f(t, z),
$$

for all $z \in K, 0<s<\delta, R<t<t+s<t_{k}$.

ii) For every $K \Subset \Omega$, for every $\epsilon>0$ and $t_{k-1}<R<S<t_{k}(1 \leq k \leq m)$, there exists $0<\delta \ll 1$ such that

$$
(1+\epsilon) f(t, z) \geq f(t+s, z)
$$

for all $z \in K, 0<s<\delta, R<t<t+s<S$.

If for every $\epsilon>0$, there exists a continuous $\epsilon$-superbarrier for (22) then there exists a unique solution to (22).

In particular, if $\left(u_{0}(z), \mu(0, z)\right)$ is admissible then (22) admits a unique solution.

Proof. By applying Theorem 4.9 and using induction, there exists a unique function $u \in C([0, T) \times \bar{\Omega})$ satisfying 
- $e^{\partial_{t} u+F(t, z, u)} \mu(t, z)=\left(d d^{c} u\right)^{n}$ in $\left(t_{k-1}, t_{k}\right) \times \Omega$ in the viscosity sense for $k=$ $1, \ldots, m$.

- $u=\varphi$ in $(0, T) \times \partial \Omega$.

- $u(0, z)=u_{0}(z)$ for every $z \in \Omega$.

Moreover, $\left(u\left(t_{k}, z\right), \mu\left(t_{k}, z\right)\right)$ is admissible for every $k=1, \ldots, m-1$. Then, by using Theorem 4.12, we get that $u$ is the unique solution to (22).

Corollary 4.14. Assume that there exist $0=t_{0}<t_{1}<\ldots<t_{m}=T$ and $f_{0}, \ldots, f_{m} \in$ $C(\Omega)$ such that

- $0 \leq f_{0} \leq f_{1} \leq \ldots \leq f_{m}$;

- $\left(u_{0}, f_{0} d V\right)$ is admissible;

- $f(t, z)=\frac{\left(t-t_{k-1}\right) f_{k}(z)+\left(t_{k}-t\right) f_{k-1}(z)}{t_{k}-t_{k-1}}$ for every $k=1, \ldots, m$ and $(t, z) \in$ $\left[t_{k-1}, t_{k}\right] \times \Omega$.

Then (2) has a unique solution.

4.3. The proof of Theorem 1.3. The first conclusion of Theorem 1.3 holds due to Remark 4.11. We now prove that admissibility is local.

Proposition 4.15. Let $g \geq 0$ be a bounded continuous function in $\Omega$ and $\nu=g d V$. Let $\phi \in P S H(\Omega) \cap C(\bar{\Omega})$. If for every $z \in \Omega$, there exists an open neighborhood $U$ of $z$ such that $(\phi, \nu)$ is admissible in $U$ then $(\phi, \nu)$ is admissible in $\Omega$.

Proof. Let $\rho \in C^{2}(\bar{\Omega}) \cap P S H(\Omega)$ such that $\left.\rho\right|_{\partial \Omega}=0,\left.\nabla \rho\right|_{\partial \Omega} \neq 0$ and $d d^{c} \rho \geq d d^{c}|z|^{2}$. For every $r>0$, we define

$$
\Omega_{r}=\{z \in \Omega \mid \rho(z)<-r\} .
$$

We will show that for all $r>0$, if $\Omega_{2 r} \neq \emptyset$ then $(\phi, \nu)$ is admissible in $\Omega_{2 r}$.

By the assumption and by the compactness of $\overline{\Omega_{2 r}}$, there exist balls $B\left(p_{1}, r_{1}\right), \ldots, B\left(p_{m}, r_{m}\right)$ such that

- $B\left(p_{k}, 2 r_{k}\right) \subset \Omega_{r}$ for all $k=1, \ldots, m$;

- $(\phi, \nu)$ is admissible in $B\left(p_{k}, 2 r_{k}\right)$ for all $k=1, \ldots, m$;

- $\overline{\Omega_{2 r}} \subset \bigcup_{k=1}^{m} B\left(p_{k}, r_{k}\right)$.

Let $\epsilon>0$. For every $k=1, \ldots, m$, there exists $C_{\epsilon, k}>0$ and $\phi_{\epsilon, k} \in C\left(B\left(p_{k}, 2 r_{k}\right)\right)$ such that $\phi \leq \phi_{\epsilon, k} \leq \phi+\epsilon$ and $\left(d d^{c} \phi_{\epsilon, k}\right)^{n} \leq e^{C_{\epsilon, k}} \nu$ in the viscosity sense in $B\left(p_{k}, 2 r_{k}\right)$.

We define

$$
\begin{gathered}
\phi_{\epsilon}(z)=\min \left\{\phi_{\epsilon, k}(z)+\frac{\epsilon\left|z-p_{k}\right|^{2}}{r_{k}^{2}}: k \in\{1, \ldots, n\}\right. \text { such that } \\
\left.\left|p_{k}-z\right|<2 r_{k}\right\}-\frac{\epsilon|z|^{2}}{\min _{0 \leq k \leq m} r_{k}^{2}} .
\end{gathered}
$$

Then $\phi_{\epsilon}$ is a continuous function in $\Omega_{2 r}$ satisfying $\phi-\frac{\epsilon \max _{\bar{\Omega}}|z|^{2}}{\min _{0 \leq k \leq m} r_{k}} \leq \phi_{\epsilon} \leq \phi+\epsilon$ and $\left(d d^{c} \phi_{\epsilon}\right)^{n} \leq e^{C_{\epsilon}} \nu$ in the viscosity sense in $\Omega_{2 r}$, where $C_{\epsilon}=\max _{1 \leq k \leq m} C_{\epsilon, k}$. Hence, $(\phi, \nu)$ is admissible in $\Omega_{2 r}$. 
Now, let $\tilde{\phi} \in C(\bar{\Omega}) \cap P S H(\Omega)$ such that $\phi=\tilde{\phi}$ in $\partial \Omega$ and $\left(d d^{c} \tilde{\phi}\right)^{n}=0$ in $\Omega$. Since $\phi, \tilde{\phi}$ are continuous, for every $\epsilon>0$, there exists $r_{1}>0$ such that $\phi \leq \tilde{\phi} \leq \phi+\frac{\epsilon}{5}$ in $\Omega \backslash \Omega_{r_{1}}$.

Let $0<r_{2}<\frac{r_{1}}{5}$. Since $(\phi, \nu)$ is admissible in $\Omega_{r_{2}}$, there exist $\phi_{\epsilon} \in C\left(\Omega_{r_{2}}\right)$ and $C_{\epsilon}>0$ such that $\phi+\frac{3 \epsilon}{5} \leq \phi_{\epsilon} \leq \phi+\frac{4 \epsilon}{5}$ and $\left(d d^{c} u_{\epsilon}\right)^{n} \leq e^{C_{\epsilon}} \mu$ in the viscosity sense in $\Omega_{r_{2}}$. We define

$$
\phi_{0, \epsilon}=\left\{\begin{array}{l}
\tilde{\phi}-\frac{\epsilon \rho}{r_{1}} \quad \text { in } \quad \Omega \backslash \Omega_{r_{2}}, \\
\min \left\{\tilde{\phi}-\frac{\epsilon \rho}{r_{1}}, \phi_{\epsilon}\right\} \quad \text { in } \quad \Omega_{r_{2}} .
\end{array}\right.
$$

Then $\phi_{0, \epsilon}$ is a continuous function in $\Omega$ satisfying $\phi \leq \phi_{0, \epsilon} \leq \phi+\epsilon$ and $\left(d d^{c} \phi_{0, \epsilon}\right)^{n} \leq$ $e^{C_{\epsilon}} \nu$ in the viscosity sense in $\Omega$. Hence, $(\phi, \nu)$ is admissible in $\Omega$.

Proposition 4.16. Let $g \geq 0$ be a bounded continuous function in $\Omega$ and $\nu=g d V$. Let $\phi \in P S H(\Omega) \cap C(\bar{\Omega})$. If $\int_{\{g=0\}}\left(d d^{c} \phi\right)_{P}^{n}=0$ then $(\phi, \nu)$ is admissible.

Proof. The problem is local by Proposition 4.15.

Let $B \Subset \Omega$ be a ball. Let $\left\{U_{j}\right\}_{j=1}^{\infty}$ be a decreasing sequence of open subsets of $B$ such that

- $\{g=0\} \cap B \subset U_{j}$ for all $j \in \mathbb{Z}^{+}$.

- $\int_{\bar{U}_{j}}\left(d d^{c} \phi\right)_{P}^{n}<\frac{1}{2^{j}}$ for all $j \in \mathbb{Z}^{+}$.

Let $\phi_{j} \in C^{\infty}(\bar{B}) \cap P S H(B)$ such that $\phi+\frac{1}{2^{j+1}} \leq \phi_{j} \leq \phi+\frac{1}{2^{j}}$ in $B$ and $\int_{U_{j}}\left(d d^{c} \phi_{j}\right)^{n}<$ $\frac{1}{2^{j+1}}$. For any $j$, we define by $\psi_{j}$ the solution of

$$
\left\{\begin{array}{l}
\psi_{j} \in C(\bar{B}) \cap P S H(B), \\
\left(d d^{c} \psi_{j}\right)_{P}^{n}=\chi_{B \backslash U_{j}}\left(d d^{c} \phi_{j}\right)^{n} \quad \text { in } B \\
\psi_{j}=\phi_{j} \quad \text { in } \quad \partial B .
\end{array}\right.
$$

Then $\psi_{j} \geq \phi_{j} \geq \phi$ and by [Xin96, Lemma 1], for any $\epsilon>0$,

$$
\lim _{j \rightarrow \infty} \operatorname{Cap}\left(\left\{\psi_{j}>\phi_{j}+\epsilon\right\}, B\right)=0,
$$

where

$$
C a p(U, \Omega)=\sup \left\{\int_{U}\left(d d^{c} w\right)^{n}: w \in P S H(\Omega), 0 \leq w \leq 1\right\},
$$

for every Borel set $U \subset \Omega$.

Hence, for every $\epsilon>0$ and $k>0$,

$$
\phi \leq\left(\limsup _{j \rightarrow \infty} \psi_{j}\right)^{*} \leq \phi_{k}+\epsilon \leq \phi+\frac{1}{2^{k}}+\epsilon .
$$

By Hartogs' lemma, $\psi_{j}$ is uniformly convergent to $\phi$ in $\bar{B}$. Moreover, $\left(\psi_{j}, \nu\right)$ is admissible in $B$ for all $j$. Hence, $(\phi, \nu)$ is admissible in $B$. Thus $(\phi, \nu)$ is admissible in $\Omega$. 
Remark 4.17. The condition " $(\phi, g d V)$ is admissible" does not imply that

$$
\int_{\{g=0\}}\left(d d^{c} \phi\right)_{P}^{n}=0 .
$$

Indeed, if $\Omega$ is the unit ball, $g=\max \left\{|z|^{2}-1 / 2,0\right\}$ and $\phi=\log \max \left\{|z|^{2}, 1 / 2\right\}$ then $(\phi, g d V)$ is admissible since $\phi_{m}=\log \max \left\{|z|^{2}, 1 / 2+1 / m\right\}$ is uniformly convergent to $\phi$ as $m \rightarrow \infty$ and $\left(\phi_{m}, g d V\right)$ is admissible for every $m>0$. But, it is clear that $\int_{\{g=0\}}\left(d d^{c} \phi\right)_{P}^{n}>0$

\section{HÖLDER CONTINUITY OF SOLUTION}

In this section we prove a Hölder regularity for the viscosity solutions to certain degenerate parabolic complex Monge-Ampère equations in smooth bounded strongly pseudoconvex domains.

Proposition 5.1. Assume that $u(t, z)$ is a viscosity solution to (2). Suppose that there exist $C>0$ and $0<\alpha<1$ such that

$$
|\varphi(t, z)-\varphi(s, z)| \leq C|t-s|^{\alpha},
$$

for all $z \in \partial \Omega, t, s \in[0, T)$. Then there exists $\tilde{C}>0$ depending on $C, n, \Omega, \sup _{[0, T) \times \Omega} f$, $\alpha$ and $\sup F(t, z, \sup \varphi)$ such that $[0, T) \times \bar{\Omega}$

$$
u(t, z)-u(s, z) \geq-\tilde{C}|t-s|^{\alpha},
$$

for all $z \in \Omega, 0 \leq s \leq t<T$.

Proof. Since $u$ is bounded, we only need to show (44) in the case $|t-s|<1$.

Let $0 \leq s_{0}<t_{0}<T$ such that $t_{0}-s_{0}=\delta<1$. Let $\rho \in P S H(\Omega) \cap C^{2}(\bar{\Omega})$ such that $\left.\rho\right|_{\partial \Omega}=0$ and $\left(d d^{c} \rho\right)^{n} \geq \mu$. Denote

$$
C_{1}=\max _{\bar{\Omega}}(-\rho), \quad C_{2}=\alpha^{-1}\left|\sup _{[0, T) \times \bar{\Omega}} F(t, z, \sup \varphi)\right|+n \sup _{(0,1)}\left(-r^{1-\alpha} \log r\right),
$$

and

$$
u_{\delta}(t, z)=u\left(s_{0}, z\right)+\delta^{\alpha} \rho-\max \left\{C, C_{2}\right\}\left(t-s_{0}\right)^{\alpha} .
$$

We have, for every $(t, z) \in\left(s_{0}, t_{0}\right) \times \Omega$,

$$
\begin{aligned}
\partial_{t} u_{\delta}(t, z)=-\frac{\alpha \max \left\{C, C_{2}\right\}}{\left(t-s_{0}\right)^{1-\alpha}} & \leq-\frac{C_{2} \alpha}{\left(t_{0}-s_{0}\right)^{1-\alpha}} \\
& \leq-\frac{\left|\sup _{[0, T) \times \bar{\Omega}} F(t, z, \sup \varphi)\right|+n \alpha \sup _{(0,1)}\left(-r^{1-\alpha} \log r\right)}{\left|\sup ^{1-\alpha} F(t, z, \sup \varphi)\right|+n \alpha\left(-\delta^{1-\alpha} \log \delta\right)} \\
& \leq-\frac{\mid[0, T) \times \bar{\Omega}}{\sup ^{1-\alpha} F(t, z, \sup \varphi) \mid} \\
& =-\frac{[0, T) \times \bar{\Omega}}{\delta^{1-\alpha}}+n \alpha \log \delta .
\end{aligned}
$$


Therefore, for each $\left(t_{1}, z_{1}\right) \in\left(s_{0}, t_{0}\right) \times \Omega$, for every upper test function $q$ for $u_{\delta}$ at $\left(t_{1}, z_{1}\right)$, we have

$$
\partial_{t} q\left(t_{1}, z_{1}\right)=\partial_{t} u_{\delta}\left(t_{1}, z_{1}\right) \leq-\frac{\left|\sup _{[0, T) \times \bar{\Omega}} F(t, z, \sup \varphi)\right|}{\delta^{1-\alpha}}+n \alpha \log \delta .
$$

Note that $q\left(t_{1}, z\right)-\delta^{\alpha} \rho(z)+\max \left\{C, C_{2}\right\}\left(t_{1}-s_{0}\right)^{\alpha}$ is a upper test function for $u_{\delta}\left(t_{1}, z\right)$ at $z_{1}$. Since $u_{\delta}\left(t_{1}, z\right)$ is plurisubharmonic [EGZ15b, Corollary 3.7], we have, by [EGZ11, Proposition 1.3],

$$
\left.d d^{c}\left(q\left(t_{1}, z\right)-\delta^{\alpha} \rho(z)\right)\right|_{z=z_{1}} \geq 0 .
$$

Then

$$
\left.d d^{c} q\left(t_{1}, z\right)\right|_{z=z_{1}} \geq\left.\delta^{\alpha} d d^{c} \rho(z)\right|_{z=z_{1}} \geq 0
$$

and

$$
\left.\left(d d^{c} q\left(t_{1}, z\right)\right)^{n}\right|_{z=z_{1}} \geq\left.\delta^{n \alpha}\left(d d^{c} \rho\right)^{n}\right|_{z=z_{1}} \geq \delta^{n \alpha} \mu\left(t_{1}, z_{1}\right) .
$$

Combining (45) and (48), we get

$$
\left(d d^{c} q\right)^{n} \geq e^{\partial_{t} q+F\left(t, z, u_{\delta}(t, z)\right)} \mu(t, z),
$$

at every $\left(t_{1}, z_{1}\right) \in\left(s_{0}, t_{0}\right) \times \Omega$.

By (47) and (49), we have

$$
\left(d d^{c} u_{\delta}\right)^{n} \geq e^{\partial_{t} u_{\delta}+F\left(t, z, u_{\delta}\right)} \mu
$$

in viscosity sense in $\left(s_{0}, t_{0}\right) \times \Omega$.

It is straightforward that $u_{\delta} \leq u$ in $\partial_{P}\left(\left(s_{0}, t_{0}\right) \times \Omega\right)$. By Theorem 2.9, we have $u_{\delta} \leq u$ in $\left(s_{0}, t_{0}\right) \times \Omega$. Hence

$$
u\left(t_{0}, z\right) \geq u_{\delta}\left(t_{0}, z\right) \geq u\left(s_{0}, z\right)-\left(C_{1}+\max \left(C, C_{2}\right)\right) \delta^{\alpha},
$$

for all $z \in \Omega$.

Thus,

$$
u(t, z) \geq u(s, z)-\left(C_{1}+\max \left(C, C_{2}\right)\right)|t-s|^{\alpha},
$$

for every $z \in \Omega$ and $0 \leq s<t<T$ with $t-s<1$. The proof is completed.

Proposition 5.2. Assume that $\mu>0$ and $u(t, z)$ is a viscosity solution to (2). Suppose that there exist $C>0,0<\alpha<1$ and $0<\beta<1 / 2$ such that

$$
|\varphi(t, z)-\varphi(s, z)| \leq C|t-s|^{\alpha},
$$

for all $z \in \partial \Omega, t, s \in[0, T)$, and

$$
\left|u_{0}(z)-u_{0}(w)\right| \leq C|z-w|^{\beta}
$$

for all $z, w \in \bar{\Omega}$. Then, there exists $\tilde{C}>0$ such that

$$
u_{0}(z)-u(t, z) \geq-\tilde{C} t^{\alpha}
$$

for all $z \in \Omega, 0 \leq t<T$.

Proof. We define on $\mathbb{C}^{n}$

$$
\tilde{u}_{0}(z)=\max _{\xi \in \bar{\Omega}}\left(u_{0}(\xi)-C|z-\xi|^{\beta}\right), z \in \mathbb{C}^{n} .
$$

Then $\tilde{u}_{0}=u_{0}$ in $\bar{\Omega}$ and

$$
\left|\tilde{u}_{0}(z)-\tilde{u}_{0}(w)\right| \leq C|z-w|^{\beta}
$$


for every $z, w \in \mathbb{C}^{n}$.

Let $\chi \in C^{\infty}\left(\mathbb{C}^{n},[0,1]\right)$ such that $\chi(z)=0$ for every $|z|>2$ and $\int_{\mathbb{C}^{n}} \chi=1$. For every $\delta>0$, we denote

$$
u_{\delta, 0}(z)=\chi_{\delta} * \tilde{u}_{0}(z)
$$

where $\chi_{\delta}(z)=\frac{1}{\delta^{2 n}} \chi\left(\frac{z}{\delta}\right)$.

Then, there exists $C_{1}>0$ depending only on $\chi$ and $C$ such that, for every $\delta>0$ and $z \in \mathbb{C}^{n}$,

$$
\left|u_{\delta, 0}(z)-\tilde{u}_{0}\right| \leq C_{1} \delta^{\beta},\left|D u_{\delta, 0}\right| \leq C_{1} \delta^{\beta-1},\left|D^{2} u_{\delta, 0}\right| \leq C_{1} \delta^{\beta-2} .
$$

Since $\mu>0$, there exists $C_{2}>0$ depending only on $C_{1}$ and $\mu$ such that

$$
\left(d d^{c} u_{\delta, 0}\right)_{+}^{n} \leq C_{2} \delta^{-2 n+n \beta} \mu .
$$

For every $0<\delta<\min \{1, T\}$, we define

$$
u_{\delta}(t, z)=u_{\delta^{\alpha / \beta}, 0}(z)+C_{1} \delta^{\alpha}+\max \left\{C, C_{3}\right\} t^{\alpha},
$$

where

$$
C_{3}=\frac{1}{\alpha}\left(\log C_{2}+\left|\inf _{[0, T) \times \bar{\Omega}} F(t, z, \inf \varphi)\right|+\frac{n \alpha(2-\beta)}{\beta} \sup _{(0,1)}\left(r^{1-\alpha} \log \frac{1}{r}\right)\right) .
$$

It is direct to check that

$$
\left(d d^{c} u_{\delta}\right)^{n} \leq e^{\partial_{t} u_{\delta}+F\left(t, z, u_{\delta}\right)} \mu
$$

in viscosity sense in $(0, \delta) \times \Omega$. Moreover, $u_{\delta} \geq u$ in $\left.\partial_{P}(0, \delta) \times \Omega\right)$. Then, by the comparison principle, $u_{\delta} \geq u$ in $(0, \delta) \times \Omega$. In particular, for every $z \in \Omega$,

$$
\begin{gathered}
u(\delta, z) \leq u_{\delta}(\delta, z)=u_{\delta^{\alpha / \beta}, 0}(z)+C_{1} \delta^{\alpha}+\max \left\{C, C_{3}\right\} \delta^{\alpha} \leq \\
u_{0}(z)+\left(2 C_{1}+\max \left\{C, C_{3}\right\}\right) \delta^{\alpha} .
\end{gathered}
$$

Since $0<\delta<\min \{1, T\}$ is arbitrary, we get

$$
u(t, z) \leq u_{0}(z)+\left(2 C_{1}+\max \left\{C, C_{3}\right\}\right) t^{\alpha},
$$

for every $(t, z) \in(0, \min \{1, T\}) \times \Omega$. Since $u$ is bounded, there exists $C_{4}>0$ depending only on $C_{1}, C_{3}, C$, sup $|u|$ such that

$$
u(t, z) \leq u_{0}(z)+C_{4} t^{\alpha},
$$

for every $(t, z) \in(0, T) \times \Omega$. The proof is completed.

Proposition 5.3. Assume that $u(t, z)$ is a viscosity solution to (2) with $\mu=d V=$ $\left(d d^{c}|z|^{2}\right)^{n}$. Suppose that there exist $C_{1}, C_{2}>0$, and $0<\beta<1 / 2$ such that

$$
\begin{aligned}
|\varphi(t, z)-\varphi(t, w)| & \leq C_{1}|z-w|^{2 \beta}, \forall z, w \in \partial \Omega, t \in[0, T) \\
\left|u_{0}(z)-u_{0}(w)\right| & \leq C_{1}|z-w|^{\beta}, \forall z, w \in \bar{\Omega},
\end{aligned}
$$

and $\forall z \in \partial \Omega, t \mapsto \varphi(t, z)-C_{2} t$ is decreasing. Then there exists $\tilde{C}>0$ such that

$$
|u(t, z)-u(t, w)| \leq \tilde{C}|z-w|^{\beta} .
$$


Proof. Let $M=\sup _{[0, T) \times \Omega} F(t, z, u(t, z))$, then $u$ satisfies

$$
\left(d d^{c} u\right)^{n} \leq e^{\partial_{t} u+M} \mu
$$

in the viscosity sense. Let $v(t, z)$ be the solution of the complex Monge-Ampère equations

$$
\left\{\begin{array}{l}
\left(d d^{c} v\right)^{n}=e^{M+C_{2}} \mu \\
v(t, z)=\varphi(t, z) \text { on } \partial \Omega
\end{array}\right.
$$

where $C_{2}$ satisfies that $\varphi(t, z)-C_{2} t$ is decreasing. Then $v(t, z)-C_{2} t$ is also the solution of

$$
\left\{\begin{array}{l}
\left(d d^{c} v\right)^{n}=e^{M+C_{2}} \mu, \\
v(t, z)=\varphi(t, z)-C_{2} t \quad \text { on } \partial \Omega .
\end{array}\right.
$$

Applying the global maximum principle of complex Monge-Ampère operator (see for example [GZ17, Corollary 3.30]) for $v(t, x)-C_{2} t$ and the fact that $\varphi(t, z)-C_{2} t$ is decreasing, we have

$$
v(t, z)-v(s, z) \leq C_{2}(t-s), \forall t \geq s .
$$

We now have $v(t, s)-C_{2} t$ is decreasing in $t$, so $v(t, z)$ converges, as $t \rightarrow 0$, to a psh function $v_{0}$ satisfying the equation

$$
\left\{\begin{array}{l}
\left(d d^{c} v_{0}\right)^{n}=e^{M+C_{2}} \mu \\
v_{0}=\varphi(0, z) \text { on } \partial \Omega .
\end{array}\right.
$$

Let $\rho \in C^{2}(\bar{\Omega}) \cap P S H(\Omega)$ such that $\rho<0$ on $\Omega,\left.\rho\right|_{\partial \Omega}=0$ and $\left(d d^{c} \rho\right)^{n} \geq \mu$. We choose $K>0$ such that $v-K(-\rho)^{\beta} \leq u_{0}$ on $\bar{\Omega}$. It follows from (53) and (52) that

$$
\left(d d^{c}\left(v-K(-\rho)^{\beta}\right)\right)^{n} \geq\left(d d^{c} v\right)^{n}=e^{C_{2}+M} \mu \geq e^{\partial_{t} v+M} \mu,
$$

in the viscosity sense. Combining with (50) and the parabolic comparison principle yields $u \geq v-K(-\rho)^{\beta}$. Moreover, we also have that $v(t, \cdot)$ is uniformly $\beta$-Hölder in $\bar{\Omega}$ (cf. [BT76, Cha15])

For the super-barrier, we use the fact that the harmonic extension $u_{\varphi}$ of $\varphi(t, z)$ majorizes $u$ from above. Moreover it follows from classical elliptic regularity that

$$
\left|u_{\varphi}(t, z)-u_{\varphi}(t, w)\right| \leq C|z-w|^{\beta}, \forall t \in[0, T] .
$$

Combining both sub/super barriers implies that there exists $B>0$ such that

$$
\forall z \in \bar{\Omega}, \forall \xi \in \partial \Omega, \quad|u(t, z)-u(t, \xi)| \leq B|z-\xi|^{\beta}, \forall t \in[0, T) .
$$

Consider $\tau \in \mathbb{C}^{n}$ small with $|\tau|<1$, the function

$$
w(t, z)=\left(1-|\tau|^{\beta}\right) u(t, z+\tau)+A_{2}|\tau|^{\beta}|z|^{2}-A_{1}|\tau|^{\beta}
$$

is defined on $\Omega_{\tau}=\{z \in \Omega \mid z+\tau \in \Omega\}$. Here we choose $A_{2}=e^{\left(C_{F}+M\right) / n}, A_{1}=$ $A_{2} \operatorname{diam}(\Omega)+|u|_{L^{\infty}}+B$, where $C_{F}$ is the Hölder constant of $F$ :

$$
|F(t, z, r)-F(t, \xi, r)| \leq C_{F}|z-\xi|^{\beta}, \forall(t, r) \in[0, T) \times\left[-\|u\|_{L^{\infty}},\|u\|_{L^{\infty}}\right] .
$$

It follows from (57) that if $z+\tau \in \partial \Omega$ or $z \in \partial \Omega$ then

$w(t, z) \leq u(t, z)-|\tau|^{\beta} u(t, z)+B\left(1-|\tau|^{\beta}\right)|\tau|^{\beta}+A_{2} \operatorname{diam}(\Omega)|\tau|^{\beta}-A_{1}|\tau|^{\beta} \leq u(t, z)$. 
We now prove that $w(t, z) \leq u(t, z)$ on $\Omega_{\tau}$. Assume by contradiction that it is not the case, then consider $U_{\tau}=\left\{(t, z) \in[0, T) \times \Omega_{\tau} \mid w(t, z)>u(t, z)\right\}$.

We will show that $w$ is a subsolution for $(2)$ on $U_{\tau}$. For any $\left(t_{0}, z_{0}\right)$ and $q$ is an upper test for $v$ at $\left(t_{0}, z_{0}\right)$, then $\tilde{q}:=\left(1-|\tau|^{\beta}\right)^{-1}\left(q(t, z)-A_{2}|\tau|^{\beta}|z|^{2}+A_{1}|\tau|^{\beta}\right.$ is also a upper test for $u(\cdot, \tau+\cdot)$ at the point $\left(t_{0}, z_{0}\right)$.

By the definition of viscosity solution $\left(d d^{c} \tilde{q}\right)^{n} \geq e^{\partial_{t} \tilde{q}+F\left(t_{0}, z_{0}, u\left(z_{0}+\tau\right)\right)} \mu$, so

$$
\partial_{t} q=\left(1-|\tau|^{\beta}\right) \partial_{t} \tilde{q} \leq\left(1-|\tau|^{\beta}\right)\left(\log \frac{\left(d d^{c} \tilde{q}\right)^{n}}{\mu}-F\left(t_{0}, z_{0}+\tau, u\left(t_{0}, z_{0}+\tau\right)\right)\right) .
$$

Combining with the concavity of log det yields, at $\left(t_{0}, z_{0}\right)$,

$$
\begin{aligned}
\log \frac{\left(d d^{c} q\right)^{n}}{\mu} & =\log \frac{\left(\left(1-|\tau|^{\beta}\right) d d^{c} \tilde{q}+|\tau|^{\beta} A_{2} d d^{c}|z|^{2}\right)^{n}}{\mu} \\
& \geq\left(1-|\tau|^{\beta}\right) \log \frac{\left(d d^{c} \tilde{q}\right)^{n}}{\mu}+|\tau|^{\beta} \log \frac{A_{2}^{n}\left(d d^{c}|z|^{2}\right)^{n}}{\mu} \\
& =\partial_{t} q+\left(1-|\tau|^{\beta}\right) F\left(t_{0}, z_{0}+\tau, u\left(t_{0}, z_{0}+\tau\right)\right)+|\tau|^{\beta} \log A_{2}^{n}
\end{aligned}
$$

here for the first inequality we use the concavity of log det and for the last equality we use the estimate for $\partial_{t} q$ above and the assumption that $\mu=\left(d d^{c}|z|^{2}\right)^{n}$.

This implies that

$$
\left(d d^{c} q\right)^{n} \geq e^{\partial_{t} q+\left(1-|\tau|^{\beta}\right) F\left(t_{0}, z_{0}+\tau, u\left(t_{0}, z_{0}+\tau\right)\right)+|\tau|^{\beta} \log A_{2}^{n}} \mu .
$$

By the monotonicity of $F$ with respect to third variable, on $U_{\tau}$ we have

$$
\begin{aligned}
F\left(t_{0}, z_{0}+\tau, u\left(t_{0}, z_{0}+\tau\right)\right) & \geq F\left(t_{0}, z_{0}+\tau,\left(1-|\tau|^{\beta}\right) u\left(t_{0}, z_{0}+\tau\right)+A_{2}|\tau|^{\beta}|z|^{2}-A_{1}|\tau|^{\beta}\right) \\
& =F\left(t_{0}, z_{0}+\tau, v\left(t_{0}, z_{0}\right)\right) \\
& \geq F\left(t_{0}, z_{0}+\tau, u\left(t_{0}, z_{0}\right)\right) .
\end{aligned}
$$

Combining this with the Hölder continuity in the second variable of $F$ and the choice of $A_{2}$, we get

$$
\left(1-|\tau|^{\beta}\right) F\left(t_{0}, z_{0}+\tau, u\left(t_{0}, z_{0}+\tau\right)\right)+|\tau|^{\beta} \log A_{2}^{n} \geq F\left(t_{0}, z_{0}, u\left(t_{0}, z_{0}\right)\right) .
$$

So it follows from (59) that

$$
\left(d d^{c} q\right)^{n} \geq e^{\partial_{t} q+F\left(t_{0}, z_{0}\right)} \mu .
$$

This implies that $v$ is a viscossity subsolution to (2) on $U_{\tau}$. Therefore the comparison principle implies that $v \leq u$ on $U_{\tau}$, and we get a contradiction. Hence $U_{\tau}$ is empty. Finally we infer that, for all $z \in \Omega$,

$$
u(t, z+\tau)+A_{2}|\tau|^{\beta}|z|^{2}-A_{1}|\tau|^{\beta} \leq u(t, z) .
$$

This implies that $u$ is Hölder in the $z$ variable as required.

Proof of Theorem 1.5. The Hölder continuity for $u$ on the $z$-variable is straightforward from Proposition 5.3. In Proposition 5.2, replacing $u_{0}$ by $u_{s}$ and using the Hölder continuity in the $z$-variable, we infer that, for $0 \leq s \leq t$,

$$
u(t, z)-u(s, z) \leq \tilde{C}|t-s|^{\alpha} .
$$

Combining with Proposition 5.1 implies the Hölder continuity of $u$ as required.

In the case where $\varphi$ is Lipschitz in $t$, by using Proposition 3.2 and Proposition 3.3 , we obtain that $u$ is locally Lipschitz in $t$ uniformly in $z$. 


\section{Convergence}

In this section, we prove that the viscosity solution of a parabolic complex MongeAmpère equation recovers the solution of the corresponding elliptic equation, extending the convergence result in [EGZ15b].

Theorem 6.1. Consider the problem (2). Assume that $T=\infty, \varphi(t, z) \rightrightarrows \varphi_{\infty}(z)$ as $t \rightarrow \infty$ and $F(t, z, r) \rightrightarrows F_{\infty}(z, r)$ in $\bar{\Omega} \times \mathbb{R}$ as $t \rightarrow \infty$, where $\rightrightarrows$ denotes the uniform convergence.

Assume that $\sup _{t \geq 0} f(t, z) \in L^{1}(\Omega)$ and $f(t, z)$ converges almost everywhere to $f_{\infty}(z) \in L^{1}(\Omega)$ as $t \rightarrow \infty$. If $(2)$ admits a solution $u$ then $u(t, z)$ converges in capacity to $u_{\infty}(z)$ as $t \rightarrow \infty$, where $u_{\infty}$ is the unique solution of the equation

$$
\left\{\begin{array}{l}
u_{\infty} \in \mathcal{F}\left(\Omega, \varphi_{\infty}\right) \\
\left(d d^{c} u_{\infty}\right)_{P}^{n}=e^{F_{\infty}\left(z, u_{\infty}\right)} f_{\infty}(z) d V(z) \quad \text { in } \quad \Omega,
\end{array}\right.
$$

where $\mathcal{F}\left(\Omega, \varphi_{\infty}\right)$ is a Cegrell class (see Definition 2.16).

Moreover, if $\sup _{t \geq 0} f(t, z) \in L^{p}(\Omega)$ for some $p>1$ then $u(t, z)$ converges uniformly to $u_{\infty}(z)$ as $t \rightarrow \infty$.

Here the uniform convergence in capacity means that, for every $\epsilon>0$, there exists an open set $U \subset \Omega$ such that

$$
\operatorname{Cap}(U, \Omega):=\sup \left\{\int_{U}\left(d d^{c} w\right)^{n}: w \in P S H(\Omega), 0 \leq w \leq 1\right\}<\epsilon,
$$

and $u(t, z)$ converges uniformly to $u_{\infty}(z)$ in $\Omega \backslash U$ as $t \rightarrow \infty$. By the countable subadditivity of capacity, this is equivalent to the following: For every $\epsilon>0$, there exist an open set $U \subset \Omega$ and $T>0$ such that $\operatorname{Cap}(U, \Omega)<\epsilon$ and $\left|u(t, z)-u_{\infty}(z)\right|<\epsilon$ for every $(t, z) \in(T, \infty) \times(\Omega \backslash U)$.

Proof. Let $1 \gg \epsilon>0$. For every $T>0$, we consider the problem

$$
\left\{\begin{array}{l}
e^{\partial_{t} w+F_{\infty}(z, w)}\left(1+\epsilon^{n+1}\right) \mu_{T}(z)=\left(d d^{c} w\right)^{n} \quad \text { in } \quad(0, \infty) \times \Omega, \\
w(t, z)=\varphi(T, z)-\epsilon \quad \text { in } \quad[0, T) \times \partial \Omega, \\
w(0, z)=u(T, z)-\epsilon \quad \text { in } \bar{\Omega},
\end{array}\right.
$$

where $\mu_{T}(z)=\sup _{t \in[T, T+1]} f(t, z) d V$. It follows from Lemma 2.15 that $\left(u(T, z), \mu_{T}(z)\right)$ is admissible for every $T$. Hence, (62) admits a unique solution $u_{T}(t, z)$.

Let $T_{1}>0$ such that

$$
\left|F(t, z, r)-F_{\infty}(z, r)\right|<\log \left(1+\epsilon^{n+1}\right),
$$

for every $(t, z, r) \in\left[T_{1}, \infty\right) \times \Omega \times \mathbb{R}$ and

$$
\left|\varphi(t, z)-\varphi_{\infty}(z)\right|<\epsilon
$$

for every $(t, z) \in\left[T_{1}, \infty\right) \times \partial \Omega$.

We will find $T_{2}>T_{1}, 0<\delta \ll 1$ and $\phi \in \mathcal{F}(\Omega)$ with $\operatorname{Cap}(\{\phi<-\epsilon\}, \Omega)=O(\epsilon)$ such that $u_{T_{2}}(t, z)+\phi$ is a subsolution to the problem

$$
\begin{cases}e^{\partial_{t} w+F_{\infty}(z, w)}\left(1+\epsilon^{n+1}\right) & \sup _{s \in\left[T_{2}, T_{2}+T^{\prime}\right]} f(s, z) d V=\left(d d^{c} w\right)^{n} \quad \text { in } \quad\left(0, T^{\prime}\right) \times \Omega, \\ w(t, z)=\varphi\left(T_{2}, z\right) & \text { in }\left[0, T^{\prime}\right) \times \partial \Omega, \\ w(0, z)=u\left(T_{2}, z\right) & \text { in } \bar{\Omega},\end{cases}
$$


and $u_{T_{2}}(t+\delta, z)-\phi+2 \epsilon$ is a supersolution to the problem (66)

$$
\begin{cases}e^{\partial_{t} w+F_{\infty}(z, w)}\left(1-\epsilon^{n+1}\right) & \inf _{s \in\left[T_{2}, T_{2}+T^{\prime}\right]} f(s, z) d V=\left(d d^{c} w\right)^{n} \quad \text { in } \quad\left(0, T^{\prime}\right) \times \Omega, \\ w(t, z)=\varphi\left(T_{2}, z\right) \quad \text { in }\left[0, T^{\prime}\right) \times \partial \Omega, & \text { in } \bar{\Omega},\end{cases}
$$

for every $T^{\prime}>\delta$.

By Proposition 5.1, there exists $\delta>0$ such that

$$
u_{T}(t+s, z) \geq u_{T}(t, z)-\epsilon,
$$

for every $t, T>0, z \in \Omega$ and $s \in[0, \delta]$. By Corollary 3.5, there exists $C_{1}>0$ such that

$$
\left|\partial_{t} u_{T}(t, z)\right| \leq C_{1},
$$

for every $T>0, z \in \Omega$ and $t \geq T+\delta$.

By Lebesgue's dominated convergence theorem, $\sup _{s \geq t} f(s, z)$ and $\inf _{s \geq t} f(s, z)$ are convergent to $f_{\infty}(z)$ in $L^{1}(\Omega)$ as $t \rightarrow \infty$. Hence,

$$
\lim _{t \rightarrow \infty} \int_{\Omega}\left|\sup _{s \geq t} f(s, z)-\inf _{s \geq t} f(s, z)\right| d V=0 .
$$

Let $T_{2}>T_{1}$ such that

$$
\int_{\Omega}\left|\sup _{s \geq T_{2}} f(s, z)-\inf _{s \geq T_{2}} f(s, z)\right| d V<\frac{e^{-C_{1}-C_{2}} \epsilon^{n+1}}{n !},
$$

where $C_{2}=\sup F\left(., ., \sup \varphi_{\infty}+\epsilon\right)$.

Let $\phi$ be the unique solution to the equation

$$
\left\{\begin{array}{l}
\phi \in \mathcal{F}(\Omega), \\
\left(d d^{c} \phi\right)_{P}^{n}=e^{C_{1}+C_{2}}\left|\left(1+\epsilon^{n+1}\right) \sup _{s \geq T_{2}} f(s, z)-\left(1-\epsilon^{n+1}\right) \inf _{s \geq T_{2}} f(s, z)\right| d V .
\end{array}\right.
$$

Then, by applying Lemma 2.7 for $u_{T_{2}}(t+\delta, z), \phi(z)$ and the equation

$$
e^{\partial_{t} w+F_{\infty}(z, w)}\left(1+\epsilon^{n+1}\right) \mu_{T_{2}}(z)=\left(d d^{c} w\right)^{n},
$$

in $\left(0, T^{\prime}\right) \times \Omega$ for all $T^{\prime}>\delta$, we get that $u_{T_{2}}(t+\delta, z)-\phi(z)+2 \epsilon$ is a supersolution to $(66)$ and $u_{T_{2}}(t, z)+\phi(z)$ is a subsolution to (65).

Note that $u\left(t+T_{2}, z\right)$ is a subsolution to (66) and a supersolution to (65). Since $u_{T_{2}}(t, z)$ is locally Lipschitz in $t$ uniformly in $z$, applying Theorem 2.9 and letting $T^{\prime} \rightarrow \infty$, we get

$$
u\left(t+T_{2}, z\right) \leq u_{T_{2}}(t+\delta, z)-\phi(z)+2 \epsilon,
$$

and

$$
u\left(t+T_{2}, z\right) \geq u_{T_{2}}(t, z)+\phi(z),
$$

for every $(t, z) \in(0, \infty) \times \Omega$.

It follows from Theorem 6.2 in [EGZ15b] that $u_{T_{2}}(t, z)$ converges uniformly to the solution $\tilde{u}$ of the equation

$$
\left\{\begin{array}{l}
\left(d d^{c} w\right)^{n}=e^{F_{\infty}(z, w)}\left(1+\epsilon^{n}\right) \mu_{T_{2}}(z) \quad \text { in } \quad \Omega, \\
w=\varphi_{\infty}-\epsilon \quad \text { in } \quad \partial \Omega .
\end{array}\right.
$$


Hence, by (71) and (72), there exists $T_{3}>0$ such that, for every $t>T_{3}$,

$$
\tilde{u}(z)-\epsilon \leq u(t, z) \leq \tilde{u}(z)-\phi(z)+3 \epsilon .
$$

It is easy to check that $\tilde{u}+\phi$ is a subsolution to (61) and $u_{\infty}+\phi-\epsilon$ is a subsolution to $(73)$. Then

$$
\tilde{u}+\phi \leq u_{\infty} \leq \tilde{u}-\phi+\epsilon .
$$

Combining (74) and (75), we get

$$
\left|u(t, z)-u_{\infty}(z)\right| \leq-2 \phi+3 \epsilon
$$

for every $t>T_{3}, z \in \Omega$.

Moreover, it follows from Proposition 3.4 in [NP09] that

$$
\operatorname{Cap}(\{\phi<-\epsilon\}, \Omega) \leq\left(1+n ! 2 C_{3} e^{C_{1}+C_{2}}\right) \epsilon,
$$

where $C_{3}=\int_{\Omega} \sup _{t>0} f(t, z) f V$.

Hence, $u(t, z)$ converges uniformly in capacity to $u_{\infty}(z)$ as $t \rightarrow \infty$.

If $\sup _{t \geq 0} f(t, z) \in L^{p}(\Omega)$ for some $p>1$ then we can choose $T_{2}$ such that

$$
\int_{\Omega}\left|\sup _{s \geq T_{2}} f(s, z)-\inf _{s \geq T_{2}} f(s, z)\right|^{p} d V<\frac{e^{-C_{1}-C_{2}} \epsilon^{n+1}}{n !} .
$$

Then, by (76) and by using Theorem 1.1 in [GKZ08] for $\phi$ and 0, we obtain the uniform convergence of $u(t, z)$ as $t \rightarrow \infty$.

\section{REFERENCES}

[Aha07] P. Ahag, A Dirichlet problem for the complex Monge-Ampère operator in $\mathcal{F}(f)$, Michigan Math. J. 55 (2007), no. 1, 123-138.

[ACCP09] P. Ahag, U. Cegrell, R. Czyz, H.-H. Pham: Monge-Ampère measures on pluripolar sets, J. Math. Pures Appl. (9) 92 (2009), no. 6, 613-627.

[Blo06] Z. Blocki: The domain of definition of the complex Monge-Ampère operator, Amer. J. Math. 128 (2006), no.2, 519-530.

[BT76] E. Bedford, B. A. Taylor, The dirichlet problem for a complex Monge-Ampère equation, Invent. Math. 37 (1976), no. 1, 1-44 .

[BT82] E. Bedford, B. A. Taylor, A new capacity for plurisubharmonic functions, Acta Math. 149 (1982), no. 1, 1-40.

[Ceg84] U. Cegrell,On the Dirichlet problem for the complex Monge-Ampère operator, Math. Z. 185, no. 2, 247-251 (1984).

[Ceg98] U. Cegrell, Pluricomplex energy, Acta Math. 180 (1998), 187217.

[Ceg04] U. Cegrell, The general definition of the complex Monge-Ampère operator, Ann. Inst. Fourier, 54 (2004), no. 1, 159-179.

[Cha15] M. Charabati, Hölder regularity for solutions to complex Monge-Ampère equations, Ann. Pol. Math., Tome 113 (2015) no. 2, pp. 109-127

[CIL92] M. G. Crandall, H. Ishii and P. L. Lions User's guide to viscosity solutions of second order partial differential equations, Bull. Amer. Math. Soc. 27 (1992), no. $1,1-67$.

[DDGHKZ] J.-P.Demailly, S.Dinew,V, Guedj, P.H.Hiep, S.Kolodziej, A.Zeriahi, Hölder continuous solutions to MongeAmpère equations, J. Eur. Math. Soc. 16 (2014), 619-647.

[DDT19] S. Dinew, H-S Do and T. D. Tô, A viscosity approach to the Dirichlet problem for degenerate complex Hessian type equations, Analysis \& PDE, 12 (2019), No. 2, 505-535. 
[EGZ11] P. Eyssidieux, V. Guedj and A. Zeriahi, Viscosity solutions to degenerate complex Monge-Ampère equations, Comm. Pure Appl. Math. 64 (2011), no. 8, 1059-1094.

[EGZ15a] _ Continuous approximation of quasiplurisubharmonic functions, Cont. Math. 644 (2015), 67-78.

[EGZ15b] _ _ Weak solutions to degenerate complex Monge-Ampère flows I, Math. Ann. 362 (2015), 931-963.

[EGZ16] _ Weak solutions to degenerate complex Monge-Ampère flows II, Adv. Math. 293 (2016), 37-80.

[EGZ18] _ Convergence of weak Kähler-Ricci flows on minimal models of positive Kodaira dimension, Comm. Math. Phys. 357 (2018), no. 3, 1179-1214.

[GKZ08] V. Guedj, S. Kolodziej and A. Zeriahi, Hölder continuous solutions to MongeAmpère equations, Bull. London Math. Soc. 40, no. 6, 1070-1080 (2008).

[GLZ1] V. Guedj, C.H .Lu, A. Zeriahi, The pluripotential Cauchy-Dirichlet problem for complex Monge-Ampère flows, arXiv:1810.02122 (2018).

[GLZ2] V. Guedj, C.H .Lu, A. Zeriahi, Pluripotential Kähler-Ricci flows, arXiv:1810.02121 (2018).

[GLZ3] V. Guedj, C.H .Lu, A. Zeriahi, Pluripotential versus viscosity solutions to complex Monge-Ampère flows, arXiv 1909.07069 (2019).

[GLZ4] V. Guedj, C.H .Lu, A. Zeriahi,Stability of solutions to complex Monge-Ampere flows, Ann. Inst. Fourier, 68 (2018), 2819-2836.

[GZ05] V. Guedj, A. Zeriahi, Intrinsic capacities on compact Kähler manifolds, J. Geom. Anal. 15, no. 4, 607-639 (2005).

[GZ17] V. Guedj and A. Zeriahi, Degenerate complex Monge-Ampère equations, EMS Tracts in Mathematics, 26. European Mathematical Society (EMS), Zrich, 2017. xxiv+472 pp. ISBN: 978-3-03719-167-5

[HL09] F.R. Harvey and H.B.Lawson, Dirichlet duality and the nonlinear Dirichlet problem, Commun. Pure Appl. Math. 62, no. 3, 396-443 (2009).

[IL90] H. Ishii, and P.L. Lions, Viscosity solutions of fully nonlinear second-order elliptic partial differential equations, J. Differential Equations 83 (1990), no. 1, 2678.

[IS13] C. Imbert, L. Silvestre: An introduction to fully nonlinear parabolic equations. An introduction to the Kähler-Ricci flow, 7-88, Lecture Notes in Math., 2086, Springer, Cham (2013).

[Kol98] S. Kołodziej, The complex Monge-Ampère equation, Acta Math. 180, (1998) no. $1,69-117$.

[Lu13] H.C Lu, Viscosity solutions to complex Hessian equations, J. Funct. Anal. 264 (2013), no. 6, 1355-1379.

[NP09] V.-K. Nguyen, H.-H. Pham: A comparison principle for the complex MongeAmpère operator in Cegrell's classes and applications, Trans. Amer. Math. Soc. 361 (2009), no. 10, 5539-5554.

[ST12] J. Song and G, Tian, Canonical measures and Kähler Ricci flow, J. Am. Math. Soc. 25, no. 2, 303-353 (2012).

[ST17] J. Song and G, Tian, The Kähler-Ricci flow through singularities, Invent. Math. 207 (2017), no. 2, 519-595.

[Tô19] T. D. Tô, Convergence of the weak Kähler-Ricci Flow on manifolds of general type, arXiv:1905.01276.

[Wal68] J. B. Walsh Continuity of Envelopes of Plurisubharmonic Functions, J. Math. Mech. 18 (1968), no. 2, 143-148.

[Wan12] Y. Wang A Viscosity Approach to the Dirichlet Problem for Complex MongeAmpère Equations, Math. Z. 272 (2012), Issue 1-2, pp 497-513.

[Xin96] Y. Xing, Continuity of the Complex Monge-Ampère Operator, Proc. Amer. Math. Soc. 124 (1996), No. 2, 457-467. 
Institute of Mathematics, Vietnam Academy of Science and Technology, 18 HoANG QuOC Viet, Hanoi, Vietnam

E-mail address: hoangson.do.vn@gmail.com, dhson@math.ac.vn

Department of Mathematics, Hanoi National University of Education, 136-Xuan Thuy, Cau Giay, Hanoi, Vietnam

E-mail address: legiang@hnue.edu.vn, legiang01@yahoo.com

Ecole Nationale de l'Aviation Civile and Institut de Mathmatiques de Toulouse (Chercheur associé), Unversité Fédérale de Toulouse, 7, Avenue Edouard Belin, FR-31055 TOulouse CEDEX

E-mail address: tat-dat.to@enac.fr, tatdat.to@gmail.com 